\title{
On MLEs in an extended multivariate linear growth curve model
}

\author{
Katarzyna Filipiak • Dietrich von Rosen
}

Received: 14 January 2010

(C) The Author(s) 2011. This article is published with open access at Springerlink.com

\begin{abstract}
In this paper the extended growth curve model is considered. The literature comprises two versions of the model. These models can be connected by one-to-one reparameterizations but since estimators are non-linear it is not obvious how to transmit properties of estimators from one model to another. Since it is only for one of the models where detailed knowledge concerning estimators is available (Kollo and von Rosen, Advanced multivariate statistics with matrices. Springer, Dordrecht, 2005) the object in this paper is therefore to present uniqueness properties and moment relations for the estimators of the second model. One aim of the paper is also to complete the results for the model presented in Kollo and von Rosen (Advanced multivariate statistics with matrices. Springer, Dordrecht, 2005). The presented proofs of uniqueness for linear combinations of estimators are valid for both models and are simplifications of proofs given in Kollo and von Rosen (Advanced multivariate statistics with matrices. Springer, Dordrecht, 2005).
\end{abstract}

Keywords Extended growth curve model - Maximum likelihood estimators · Moments · Estimability

\section{Introduction}

In experiments, in which more than one characteristic of every treatment is measured, multivariate linear models may be applied. A well known and interesting multivariate

\footnotetext{
K. Filipiak ( $\varangle)$

Department of Mathematical and Statistical Methods, Poznań University of Life Sciences,

Wojska Polskiego 28, 60637 Poznań, Poland

e-mail: kasfil@up.poznan.pl

D. von Rosen

Energy and Technology, Swedish University of Agricultural Sciences, 75007 Uppsala, Sweden
} 
linear model is the growth curve model (GCM) due to Potthof and Roy (1964) which belongs to the curved exponential family. Many results and references can be found in Kollo and von Rosen (2005) [Chapter 4]. Among others explicit maximum likelihood estimators (MLE), estimability conditions, moments and approximative distributions of the estimators are available.

Markiewicz and Szczepańska (2007) considered the GCM with additional nuisance parameters. They determined estimators of the parameters of interest as well as presented the first and second order moments of this estimator. Kiefer optimal designs and relations between optimality under univariate and multivariate models were given. Moreover, the GCM with two nuisance parameters was considered in Filipiak et al. (2009). The authors gave estimators of two matrices of parameters and obtained appropriate moment relations to determine Kiefer optimal designs.

Consider a linear model

$$
\mathbf{y}=\mathbf{A}_{1} \boldsymbol{\beta}_{1}+\mathbf{A}_{2} \boldsymbol{\beta}_{2}+\mathbf{A}_{3} \boldsymbol{\beta}_{3}+\boldsymbol{\epsilon}
$$

where $\mathbf{A}_{i} \in \mathbb{R}^{n \times m_{i}}, \quad i=1,2,3$, are known design matrices and $\mathbf{y} \in \mathbb{R}^{n}$ is an observable random vector, which depends linearly on several parameters. The model in (1) represents measurements on a single response variable $y$. Here $\boldsymbol{\beta}_{i} \in \mathbb{R}^{m_{i}}, i=$ $1,2,3$, are vectors of parameters, and $\epsilon \in \mathbb{R}^{n}$ is a vector of normally distributed random errors with expectation $E[\boldsymbol{\epsilon}]=\mathbf{0}$, and dispersion matrix $D[\boldsymbol{\epsilon}]=\sigma^{2} \mathbf{I}_{n}$, where $\sigma^{2}$ is unknown constant and $\mathbf{I}_{n}$ is the identity matrix of order $n$.

If we are measuring $p$ response variables on each sampling unit we can extend (1) and consider the following multivariate linear model

$$
\mathbf{Y}=\mathbf{A}_{1} \mathbf{B}_{1} \mathbf{C}_{1}+\mathbf{A}_{2} \mathbf{B}_{2} \mathbf{C}_{2}+\mathbf{A}_{3} \mathbf{B}_{3} \mathbf{C}_{3}+\mathbf{E}
$$

where in addition to $\mathbf{A}_{i}$ the matrices $\mathbf{C}_{i} \in \mathbb{R}^{q_{i} \times p}, i=1,2,3$, are known. The matrix $\mathbf{Y} \in \mathbb{R}^{n \times p}$ is an observations matrix and $\mathbf{B}_{i} \in \mathbb{R}^{m_{i} \times q_{i}}, i=1,2,3$, are matrices of unknown parameters. The matrix $\mathbf{E} \in \mathbb{R}^{n \times p}$ is a matrix of random errors, normally distributed, with expectation $E[\mathbf{E}]=\mathbf{0}$ and with dispersion matrix $D[\mathbf{E}]=$ $D[\operatorname{vec}(\mathbf{E})]=\boldsymbol{\Sigma} \otimes \mathbf{I}_{n}$, where $\boldsymbol{\Sigma} \in \mathbb{R}_{p}^{>}$is an unknown positive definite matrix, $\operatorname{vec}(\mathbf{E})$ denotes the vector in $\mathbb{R}^{p n}$ formed by putting the columns of $\mathbf{E} \in \mathbb{R}^{n \times p}$ under each other, starting from the left, and $\otimes$ denotes the Kronecker product. The matrices $\mathbf{A}_{i}$ (between-individuals design matrices) are used to design the experiment, i.e., lay out treatments in an appropriate way, whereas the $\mathbf{C}_{i}$ matrices (within-individuals design matrices) are used to describe the relation between the response variables.

The model in (2) will be called extended growth curve model (EGCM). As seen it is a generalized version of the GCM, i.e., $E[\mathbf{Y}]=\mathbf{A}_{1} \mathbf{B}_{1} \mathbf{C}_{1}$, and is sometimes termed sums of profiles model (see Verbyla and Venables 1988). The model may be viewed as a multivariate seemingly unrelated regression (SUR) model. The maximum likelihood equations can not be explicitly solved for such a model. However, if the nested subspace condition $\mathcal{R}\left(\mathbf{C}_{3}^{\prime}\right) \subseteq \mathcal{R}\left(\mathbf{C}_{2}^{\prime}\right) \subseteq \mathcal{R}\left(\mathbf{C}_{1}^{\prime}\right)$ or $\mathcal{R}\left(\mathbf{A}_{3}\right) \subseteq \mathcal{R}\left(\mathbf{A}_{2}\right) \subseteq \mathcal{R}\left(\mathbf{A}_{1}\right)$ hold, than it is possible to solve maximum likelihood equations and find explicit maximum likelihood estimators of unknown parameters. The above conditions lead to different parameterizations. However, it is only for one of them where a lot of detailed 
knowledge such as uniqueness conditions for MLEs, moments and asymptotics has been presented (e.g. Kollo and von Rosen 2005 [Chapter 4]: observe that the roles of $\mathbf{A}_{i}$ and $\mathbf{C}_{i}$ in this work are interchanged). When discussing Kiefer optimality, unfortunately, we need results for the estimators of parameters in the other parameterization. If the nested condition is not fulfilled, special structures of $\boldsymbol{\Sigma}$ may be considered (see e.g. Andersson and Perlman 1993, 1998).

In the subsequent we are going to refer to the two models as Model I and Model II $(\mathcal{R}(\bullet)$ denotes the column space).

Definition 1 Let all matrices be the same as in (2).

Model I:

$$
\mathbf{Y}=\mathbf{A}_{1} \mathbf{B}_{1} \mathbf{C}_{1}+\mathbf{A}_{2} \mathbf{B}_{2} \mathbf{C}_{2}+\mathbf{A}_{3} \mathbf{B}_{3} \mathbf{C}_{3}+\mathbf{E}, \quad \mathcal{R}\left(\mathbf{C}_{3}^{\prime}\right) \subseteq \mathcal{R}\left(\mathbf{C}_{2}^{\prime}\right) \subseteq \mathcal{R}\left(\mathbf{C}_{1}^{\prime}\right)
$$

Model II:

$$
\mathbf{Y}=\mathbf{A}_{1} \mathbf{B}_{1} \mathbf{C}_{1}+\mathbf{A}_{2} \mathbf{B}_{2} \mathbf{C}_{2}+\mathbf{A}_{3} \mathbf{B}_{3} \mathbf{C}_{3}+\mathbf{E}, \quad \mathcal{R}\left(\mathbf{A}_{3}\right) \subseteq \mathcal{R}\left(\mathbf{A}_{2}\right) \subseteq \mathcal{R}\left(\mathbf{A}_{1}\right)
$$

It will now be shown that Model I and Model II indeed are equivalent, i.e., via reparameterizations one can derive Model I from Model II or vice versa. From (3) it follows that there exist matrices $\mathbf{H}_{1}$ and $\mathbf{H}_{2}$ such that

$$
\mathcal{R}\left(\mathbf{C}_{1}^{\prime}\right)=\mathcal{R}\left(\mathbf{C}_{2}^{\prime}\right) \boxplus \mathcal{R}\left(\mathbf{H}_{1}^{\prime}\right), \quad \mathcal{R}\left(\mathbf{C}_{2}^{\prime}\right)=\mathcal{R}\left(\mathbf{C}_{3}^{\prime}\right) \boxplus \mathcal{R}\left(\mathbf{H}_{2}^{\prime}\right),
$$

where $\boxplus$ denotes the orthogonal sum of subspaces. Let $\boldsymbol{\Theta}_{1}=\left(\boldsymbol{\Theta}_{11}: \boldsymbol{\Theta}_{12}: \boldsymbol{\Theta}_{13}\right)$ and $\boldsymbol{\Theta}_{2}=\left(\boldsymbol{\Theta}_{21}: \boldsymbol{\Theta}_{22}\right)$. Then, Model I is equivalent to

$$
\begin{aligned}
& \mathbf{Y}=\left(\mathbf{A}_{1}: \mathbf{A}_{2}: \mathbf{A}_{3}\right)\left(\boldsymbol{\Theta}_{11}^{\prime}: \boldsymbol{\Theta}_{21}^{\prime}: \mathbf{B}_{3}^{\prime}\right)^{\prime} \mathbf{C}_{3} \\
&+\left(\mathbf{A}_{1}: \mathbf{A}_{2}\right)\left(\boldsymbol{\Theta}_{12}^{\prime}: \boldsymbol{\Theta}_{22}^{\prime}\right)^{\prime} \mathbf{H}_{2}+\mathbf{A}_{1} \boldsymbol{\Theta}_{13} \mathbf{H}_{1}+\mathbf{E}
\end{aligned}
$$

which according to Definition 1 is of Model II type. The main problem is that because of non-linearity of estimators it is not obvious how to transmit properties of estimators from one model to the other, in particular moment relations. This will become clear when explicit estimators are presented. Moreover, since the covariance between the estimators of $\mathbf{B}_{i}$ and $\mathbf{B}_{j}$ has not been derived in Model II it is obvious that we can not obtain the dispersion for $\mathbf{B}_{i}$ in Model I by using results from Model II.

In this paper we are mainly interested in the EGCM model with the range condition on the within-individuals design matrices, i.e., Model I. The reason for this is that in models connected to experimental designs the column spaces of between-individuals design matrices should be disjoint or, the intersection should be as small as possible (cf. regression models, interference models). For example, in block designs, only designs for that the common space of design matrices is the space spanned by the vector of ones are connected (for more details see e.g. Filipiak and Różański 2009). 
The main goal of this paper is to present results for Model I and put them into relation with results for Model II. For Model I we give estimators of the unknown parameters as well as moments of these estimators. Conditions for uniqueness of the estimators will also be given. It is worth noting, that the results are similar for both models, although the methods of deriving them differ. For example, to calculate the second order moments of estimators of unknown parameters in Model I, which are useful in determining Kiefer optimal designs (see e.g. Filipiak et al. 2009), the properties of multivariate beta distribution and its inverse must be used, while in Model II it was enough to apply the properties of Wishart distribution. Because the moments are difficult to obtain rather many details will be given, which burden the presentation but seem to be unavoidable.

Throughout the paper we use the following notation. Let $\mathbf{P}_{X}=\mathbf{X}\left(\mathbf{X}^{\prime} \mathbf{X}\right)^{-} \mathbf{X}^{\prime}$ and $\mathbf{Q}_{X}=\mathbf{I}_{m}-\mathbf{P}_{X}$ denote the orthogonal projectors on the column space $\mathcal{R}(\mathbf{X})$ and its orthocomplement, respectively. Moreover, $\mathbf{X}^{-}$denotes an arbitrary generalized inverse of the matrix $\mathbf{X}$ and $\mathbf{X}^{o}$ is any matrix spanning $\mathcal{R}(\mathbf{X})^{\perp}$. For a positive definite $\mathbf{B}$ we denote $\mathbf{P}_{X ; B}=\mathbf{X}\left(\mathbf{X}^{\prime} \mathbf{B} \mathbf{X}\right)^{-} \mathbf{X}^{\prime} \mathbf{B}$ and $\mathbf{Q}_{X ; B}=\mathbf{I}-\mathbf{P}_{X ; B}$. Since $\mathbf{P}_{B^{-1 / 2} X^{o}}=\mathbf{Q}_{B^{1 / 2} X}(\mathrm{cf}$. Markiewicz 2001; Kollo and von Rosen 2005) it follows that $\mathbf{I}=\mathbf{P}_{X ; B}+\mathbf{P}_{X^{o} ; B^{-1}}^{\prime}$ which is equivalent to

$$
\mathbf{B}=\mathbf{B} \mathbf{X}\left(\mathbf{X}^{\prime} \mathbf{B} \mathbf{X}\right)^{-} \mathbf{X}^{\prime} \mathbf{B}+\mathbf{X}^{o}\left(\mathbf{X}^{o^{\prime}} \mathbf{B}^{-1} \mathbf{X}^{o}\right)^{-} \mathbf{X}^{o^{\prime}}
$$

a formula which often will be utilized in this paper. We use $\operatorname{rank} \mathbf{X}$ and $\operatorname{tr} \mathbf{X}$ to denote the rank and the trace of $\mathbf{X}$, respectively. Moreover, sometimes it is written $(\mathbf{A})()^{\prime}$ instead of $(\mathbf{A})(\mathbf{A})^{\prime}$.

The Introduction is ended by presenting an example which illustrates Model I. Example of Model II may be found in Kollo and von Rosen (2005) [Example 4.1.2, p. 374].

Example 1 Consider an agricultural experiment. Suppose it is desired to compare the yield of $v$ different varieties of grain (treatments). It is likely that there is an interaction between the environment (type of soil, rainfall, drainage, etc.) and the variety of grain which will alter the yields. So, $b$ blocks [sets of experimental plots (units)] are chosen in which the environment is fairly consistent throughout the block; R. A. Fisher and F. Yates, early 1930's.

Let $n$ experimental units (plots) because of extraneous variability be divided into $b$ blocks each of size $k$ where the blocks consist of homogeneous units. Let $v$ treatments be applied to the units so that each unit receives one treatment. The treatment which is applied to unit $j$ in block $i$ is determined by the design $d$. In each block the effect of the treatments applied to each unit is measured by a random variable $\mathbf{y}$.

Assume, the response on a given plot may be affected by treatments on neighboring plots as well as by the treatment applied to that plot. Consider experiments with a onedimensional arrangement of plots in each block, and for which the treatments have different left and right neighbor interference effects. In the literature circular experiments (Druilhet 1999) and experiments without border plots (Kunert and Martin 2000) have been studied. 
Suppose that a linear model associated with a design $d$ has the form

$$
\mathbf{y}=\mathbf{A}_{1, d} \boldsymbol{\beta}_{1}+\mathbf{A}_{2, d} \boldsymbol{\beta}_{2}+\mathbf{A}_{3} \boldsymbol{\beta}_{3}+\boldsymbol{\epsilon},
$$

where $\boldsymbol{\beta}_{i}, i=1,2,3$, are the unknown vectors of treatment effects, neighbor effects, and block effects, respectively, and $\epsilon$ is the vector of random errors. The matrix $\mathbf{A}_{1, d} \in$ $\mathbb{R}^{n \times v}$ depends on the design and it is a binary matrix which satisfies $\mathbf{A}_{1, d} \mathbf{1}_{v}=\mathbf{1}_{n}$. The matrix $\mathbf{A}_{2, d}=\left(\left(\mathbf{I}_{b} \otimes \mathbf{H}\right) \mathbf{A}_{1, d}:\left(\mathbf{I}_{b} \otimes \mathbf{H}^{\prime}\right) \mathbf{A}_{1, d}\right)$, is a known matrix of neighbor effects, where either

$$
\mathbf{H}=\left(\begin{array}{cc}
\mathbf{0}_{k-1}^{\prime} & 1 \\
\mathbf{I}_{k-1} & \mathbf{0}_{k-1}
\end{array}\right) \quad \text { or } \quad \mathbf{H}=\left(\begin{array}{cc}
\mathbf{0}_{k-1}^{\prime} & 0 \\
\mathbf{I}_{k-1} & \mathbf{0}_{k-1}
\end{array}\right)
$$

for the circular designs and for the designs without border plots, respectively $\left(\mathbf{0}_{k-1}\right.$ is a $k-1$ dimensional vector of zeros). The matrix $\mathbf{A}_{3}=\mathbf{I}_{b} \otimes \mathbf{1}_{k}$ is the design matrix of block effects.

In the literature such a model is called an interference model with neighbor effects; see e.g. Druilhet (1999); Kunert and Martin (2000).

Case 1 Assume, we are interested in measuring $p$ characteristics of every treatment from $n$ experimental units. Then, we have the following extension of the interference model:

$$
\mathbf{Y}=\mathbf{A}_{1, d} \mathbf{B}_{1} \mathbf{C}_{1}+\mathbf{A}_{2, d} \mathbf{B}_{2} \mathbf{C}_{2}+\mathbf{A}_{3} \mathbf{B}_{3} \mathbf{C}_{3}+\mathbf{E},
$$

where $\mathbf{Y} \in \mathbb{R}^{n \times p}$ is the matrix of observations, $\mathbf{B}_{i}, \quad i=1,2,3$, are the unknown matrices of treatment, neighbor and block effects, respectively, and $\mathbf{C}_{i}, i=1,2,3$, are the restriction matrices, i.e., the between-individual design matrices.

Assume, that in the experiment there is no left- and right-neighbor effect for the last characteristic and there is no block effect for the last two characteristics. Then, $\mathbf{C}_{1}=\mathbf{I}_{p}, \mathbf{C}_{2}=\left(\mathbf{I}_{p-1}, \mathbf{0}_{p-1}\right)$ and $\mathbf{C}_{3}=\left(\mathbf{I}_{p-2}, \mathbf{0}_{p-2}, \mathbf{0}_{p-2}\right)$ and we obtain Model I. Such a situation may occur for example in experiments with oil plants (sunflowers, rape), where the neighbor effects are significant for morphological characteristics (height, yield), but not for instance the oil content in plant. The block effects may be significant for the yield, but not for some physiological characteristics (compounds content in grain, genetic characteristics).

Case 2 Consider an experiment, that some individuals are observed repeatedly in $p$ time points. Then, we have the following extension of the interference model:

$$
\mathbf{Y}=\mathbf{A}_{1, d} \mathbf{B}_{1} \mathbf{C}_{1}+\mathbf{A}_{2, d} \mathbf{B}_{2} \mathbf{C}_{2}+\mathbf{A}_{3} \mathbf{B}_{3} \mathbf{C}_{3}+\mathbf{E},
$$

where $\mathbf{Y} \in \mathbb{R}^{n \times p}$ is the matrix of observations, $\mathbf{B}_{i}, \quad i=1,2,3$, are the unknown matrices of treatment, neighbor and block effects, respectively, and $\mathbf{C}_{i}, i=1,2,3$, are the restriction matrices.

Assume, that there is polynomial regression dependence between treatment effects (polynomial of order $q_{1}$ ) and polynomial regression dependence between neighbor 
effects (polynomial of order $q_{2}, q_{2} \leq q_{1}$ ), while block effects are the same for every time point. Then $\mathbf{C}_{i}, i=1,2$, are Vandermonde matrices from $\mathbb{R}^{q_{i} \times p}$ and $\mathbf{C}_{3}=\mathbf{J}_{q_{3} \times p}$. Again we obtain Model I.

It is also worth noting that the multivariate extension of the standard cross-over model with carry-over effects (see e.g. Kunert 1983) may be formulated as Model I.

\section{Maximum likelihood estimators}

Throughout the paper we consider maximum likelihood estimators of unknown parameters in Model I as well as Model II. For Model I the results are given partially in Filipiak et al. (2009).

Theorem 1 In Model I the maximum likelihood estimators of the parameters equal

$$
\begin{aligned}
\widehat{\mathbf{B}}_{1}= & \left(\mathbf{A}_{1}^{\prime} \mathbf{A}_{1}\right)^{-} \mathbf{A}_{1}^{\prime}\left(\mathbf{Y}-\mathbf{A}_{2} \widehat{\mathbf{B}}_{2} \mathbf{C}_{2}-\mathbf{A}_{3} \widehat{\mathbf{B}}_{3} \mathbf{C}_{3}\right) \mathbf{S}_{3}^{-1} \mathbf{C}_{1}^{\prime}\left(\mathbf{C}_{1} \mathbf{S}_{3}^{-1} \mathbf{C}_{1}^{\prime}\right)^{-} \\
& +\left(\mathbf{A}_{1}^{\prime}\right)^{o} \mathbf{Z}_{11} \mathbf{C}_{1}^{\prime}+\mathbf{A}_{1}^{\prime} \mathbf{Z}_{12} \mathbf{C}_{1}^{o^{\prime}}, \\
\widehat{\mathbf{B}}_{2}= & \left(\mathbf{A}_{2}^{\prime} \mathbf{Q}_{A_{1}} \mathbf{A}_{2}\right)^{-} \mathbf{A}_{2}^{\prime} \mathbf{Q}_{A_{1}}\left(\mathbf{Y}-\mathbf{A}_{3} \widehat{\mathbf{B}}_{3} \mathbf{C}_{3}\right) \mathbf{S}_{2}^{-1} \mathbf{C}_{2}^{\prime}\left(\mathbf{C}_{2} \mathbf{S}_{2}^{-1} \mathbf{C}_{2}^{\prime}\right)^{-} \\
& +\left(\mathbf{A}_{2}^{\prime} \mathbf{Q}_{A_{1}}\right)^{o} \mathbf{Z}_{21} \mathbf{C}_{2}^{\prime}+\mathbf{A}_{2}^{\prime} \mathbf{Q}_{A_{1}} \mathbf{Z}_{22} \mathbf{C}_{2}^{o^{\prime}}, \\
\widehat{\mathbf{B}}_{3}=\left(\mathbf{A}_{3}^{\prime} \mathbf{Q}_{\left(A_{1}: A_{2}\right)} \mathbf{A}_{3}\right)^{-} \mathbf{A}_{3}^{\prime} \mathbf{Q}_{\left(A_{1}: A_{2}\right)} \mathbf{Y} \mathbf{S}_{1}^{-1} \mathbf{C}_{3}^{\prime}\left(\mathbf{C}_{3} \mathbf{S}_{1}^{-1} \mathbf{C}_{3}^{\prime}\right)^{-} & \\
& +\left(\mathbf{A}_{3}^{\prime} \mathbf{Q}_{\left(A_{1}: A_{2}\right)}\right)^{o} \mathbf{Z}_{31} \mathbf{C}_{3}^{\prime}+\mathbf{A}_{3}^{\prime} \mathbf{Q}_{\left(A_{1}: A_{2}\right)} \mathbf{Z}_{32} \mathbf{C}_{3}^{o^{\prime}},
\end{aligned}
$$

where

$$
\begin{aligned}
& \mathbf{S}_{1}=\mathbf{Y}^{\prime} \mathbf{Q}_{\left(A_{1}: A_{2}: A_{3}\right)} \mathbf{Y}, \quad \mathbf{S}_{2}=\mathbf{S}_{1}+\mathbf{Q}_{C_{3}^{\prime} ; S_{1}^{-1}} \mathbf{Y}^{\prime} \mathbf{P}_{Q_{\left(A_{1}: A_{2}\right)} A_{3}} \mathbf{Y} \mathbf{Q}_{C_{3}^{\prime} ; S_{1}^{-1}}^{\prime}, \\
& \mathbf{S}_{3}=\mathbf{S}_{2}+\mathbf{Q}_{C_{2}^{\prime} ; S_{2}^{-1}} \mathbf{Y}^{\prime} \mathbf{P}_{Q_{A_{1}} A_{2}} \mathbf{Y} \mathbf{Q}_{C_{2}^{\prime} ; S_{2}^{-1}}^{\prime},
\end{aligned}
$$

and $\mathbf{Z}_{i j}, \quad i=1,2,3, j=1,2$, are arbitrary matrices. The ML-estimator of the dispersion matrix can be written

$$
n \widehat{\mathbf{\Sigma}}=\left(\mathbf{Y}-\mathbf{A}_{1} \widehat{\mathbf{B}}_{1} \mathbf{C}_{1}-\mathbf{A}_{2} \widehat{\mathbf{B}}_{2} \mathbf{C}_{2}-\mathbf{A}_{3} \widehat{\mathbf{B}}_{3} \mathbf{C}_{3}\right)^{\prime}()=\mathbf{S}_{3}+\mathbf{Q}_{C_{1}^{\prime} ; S_{3}^{-1}} \mathbf{Y}^{\prime} \mathbf{P}_{A_{1}} \mathbf{Y} \mathbf{Q}_{C_{1}^{\prime} ; S_{3}^{-1}} .
$$

Theorem 2 (Kollo and von Rosen 2005) In Model II the maximum likelihood estimators of the parameters equal

$$
\begin{aligned}
\widehat{\mathbf{B}}_{1}= & \left(\mathbf{A}_{1}^{\prime} \mathbf{A}_{1}\right)^{-} \mathbf{A}_{1}^{\prime}\left(\mathbf{Y}-\mathbf{A}_{2} \widehat{\mathbf{B}}_{2} \mathbf{C}_{2}-\mathbf{A}_{3} \widehat{\mathbf{B}}_{3} \mathbf{C}_{3}\right) \mathbf{S}_{1}^{-1} \mathbf{C}_{1}^{\prime}\left(\mathbf{C}_{1} \mathbf{S}_{1}^{-1} \mathbf{C}_{1}^{\prime}\right)^{-} \\
& +\mathbf{Z}_{11} \mathbf{C}_{1}^{o^{\prime}}+\mathbf{A}_{1}^{\prime o} \mathbf{Z}_{12} \mathbf{C}_{1}^{\prime}, \\
\widehat{\mathbf{B}}_{2}= & \left(\mathbf{A}_{2}^{\prime} \mathbf{A}_{2}\right)^{-} \mathbf{A}_{2}^{\prime}\left(\mathbf{Y}-\mathbf{A}_{3} \widehat{\mathbf{B}}_{3} \mathbf{C}_{3}\right) \mathbf{S}_{2}^{-1} \mathbf{Q}_{C_{1}^{\prime} ; S_{1}^{-1}} \mathbf{C}_{2}^{\prime}\left(\mathbf{C}_{2} \mathbf{Q}_{C_{1}^{\prime} ; S_{1}^{-1}}^{\prime} \mathbf{S}_{2}^{-1} \mathbf{Q}_{C_{1}^{\prime} ; S_{1}^{-1}} \mathbf{C}_{2}^{\prime}\right)^{-} \\
& \quad+\mathbf{Z}_{21}\left(\mathbf{C}_{2} \mathbf{Q}_{C_{1}^{\prime} ; S_{1}^{-1}}^{\prime o^{\prime}}+\mathbf{A}_{2}^{\prime o} \mathbf{Z}_{22} \mathbf{Q}_{C_{1}^{\prime} ; S_{1}^{-1}} \mathbf{C}_{2}^{\prime},\right. \\
\widehat{\mathbf{B}}_{3}= & \left(\mathbf{A}_{3}^{\prime} \mathbf{A}_{3}\right)^{-} \mathbf{A}_{3}^{\prime} \mathbf{Y} \mathbf{S}_{3}^{-1} \mathbf{P}_{3} \mathbf{C}_{3}^{\prime}\left(\mathbf{C}_{3} \mathbf{P}_{3}^{\prime} \mathbf{S}_{3}^{-1} \mathbf{P}_{3} \mathbf{C}_{3}^{\prime}\right)^{-}+\mathbf{Z}_{31}\left(\mathbf{C}_{3} \mathbf{P}_{3}^{\prime}\right)^{o^{\prime}}+\mathbf{A}_{3}^{o o} \mathbf{Z}_{32} \mathbf{P}_{3} \mathbf{C}_{3}^{\prime},
\end{aligned}
$$


where

$$
\begin{aligned}
& \mathbf{S}_{1}=\mathbf{Y}^{\prime} \mathbf{Q}_{A_{1}} \mathbf{Y}, \quad \mathbf{S}_{2}=\mathbf{S}_{1}+\mathbf{Q}_{C_{1}^{\prime} ; S_{1}^{-1}} \mathbf{Y}^{\prime} \mathbf{P}_{A_{1}} \mathbf{Q}_{A_{2}} \mathbf{P}_{A_{1}} \mathbf{Y} \mathbf{Q}_{C_{1}^{\prime} ; S_{1}^{-1}}^{\prime}, \\
& \mathbf{S}_{3}=\mathbf{S}_{2}+\mathbf{P}_{3} \mathbf{Y}^{\prime} \mathbf{P}_{A_{1}} \mathbf{Q}_{A_{3}} \mathbf{P}_{A_{1}} \mathbf{Y} \mathbf{P}_{3}^{\prime},
\end{aligned}
$$

and $\mathbf{Z}_{i j}, i=1,2,3, j=1,2$ are arbitrary matrices, and

$$
n \widehat{\boldsymbol{\Sigma}}=\left(\mathbf{Y}-\mathbf{A}_{1} \widehat{\mathbf{B}}_{1} \mathbf{C}_{1}-\mathbf{A}_{2} \widehat{\mathbf{B}}_{2} \mathbf{C}_{2}-\mathbf{A}_{3} \widehat{\mathbf{B}}_{3} \mathbf{C}_{3}\right)^{\prime}()=\mathbf{S}_{3}+\mathbf{P}_{4} \mathbf{Y}^{\prime} \mathbf{P}_{A_{3}} \mathbf{Y} \mathbf{P}_{4}^{\prime},
$$

with $\mathbf{P}_{i}=\mathbf{U}_{i-1} \times \cdots \times \mathbf{U}_{1}, i=3,4, \mathbf{U}_{j}=\mathbf{Q}_{P_{j} C_{j}^{\prime} ; S_{j}^{-1}}, j=1,2,3$.

Both Theorems 1 and 2 can be obtained by solving the following likelihood equations:

$$
\begin{aligned}
\mathbf{0} & =\mathbf{A}_{1}^{\prime}\left(\mathbf{Y}-\mathbf{A}_{1} \mathbf{B}_{1} \mathbf{C}_{1}-\mathbf{A}_{2} \mathbf{B}_{2} \mathbf{C}_{2}-\mathbf{A}_{3} \mathbf{B}_{3} \mathbf{C}_{3}\right) \boldsymbol{\Sigma}^{-1} \mathbf{C}_{1}^{\prime}, \\
\mathbf{0} & =\mathbf{A}_{2}^{\prime}\left(\mathbf{Y}-\mathbf{A}_{1} \mathbf{B}_{1} \mathbf{C}_{1}-\mathbf{A}_{2} \mathbf{B}_{2} \mathbf{C}_{2}-\mathbf{A}_{3} \mathbf{B}_{3} \mathbf{C}_{3}\right) \boldsymbol{\Sigma}^{-1} \mathbf{C}_{2}^{\prime}, \\
\mathbf{0} & =\mathbf{A}_{3}^{\prime}\left(\mathbf{Y}-\mathbf{A}_{1} \mathbf{B}_{1} \mathbf{C}_{1}-\mathbf{A}_{2} \mathbf{B}_{2} \mathbf{C}_{2}-\mathbf{A}_{3} \mathbf{B}_{3} \mathbf{C}_{3}\right) \boldsymbol{\Sigma}^{-1} \mathbf{C}_{3}^{\prime}, \\
n \boldsymbol{\Sigma} & =\left(\mathbf{Y}-\mathbf{A}_{1} \mathbf{B}_{1} \mathbf{C}_{1}-\mathbf{A}_{2} \mathbf{B}_{2} \mathbf{C}_{2}-\mathbf{A}_{3} \mathbf{B}_{3} \mathbf{C}_{3}\right)^{\prime}() .
\end{aligned}
$$

\section{Uniqueness conditions for the MLEs}

Consider the model

$$
\mathbf{y}=\mathbf{X} \boldsymbol{\beta}+\mathbf{Z} \boldsymbol{\gamma}+\boldsymbol{\epsilon}, \quad E(\boldsymbol{\epsilon})=\mathbf{0}, \quad \operatorname{Cov}(\boldsymbol{\epsilon})=\sigma^{2} \boldsymbol{\Sigma},
$$

where $\Sigma$ is known. It is known that the least squares estimator of a linear function of the parameter vector $\boldsymbol{\beta}$, say $\mathbf{p}^{\prime} \widehat{\boldsymbol{\beta}}$, is unique if and only if $\mathbf{p}^{\prime} \boldsymbol{\beta}$ is estimable. The estimability condition may be expressed as

$$
\mathbf{p} \in \mathcal{R}\left(\mathbf{X}^{\prime} \mathbf{Q}_{Z}\right)
$$

see e.g. Baksalary (1984). Using the "vec" operator and then considering linear spaces generated by Kronecker products, which indeed are tensor spaces it can be seen that under the multivariate model with nuisance parameters and unknown $\Sigma$, i.e.,

$$
\mathbf{Y}=\mathbf{A}_{1} \boldsymbol{\Xi} \mathbf{P}_{1}+\mathbf{A}_{2} \boldsymbol{\Theta} \mathbf{P}_{2}+\mathbf{E},
$$

the estimability condition for $\mathbf{K} \mathbf{\Xi} \mathbf{L}$ can be written

$$
\mathcal{R}\left(\mathbf{L} \otimes \mathbf{K}^{\prime}\right) \subset \mathcal{R}\left(\left(\mathbf{P}_{1} \otimes \mathbf{A}_{1}^{\prime}\right) \mathbf{Q}_{P_{2}^{\prime} \otimes A_{2}}\right) ;
$$

for more details see e.g. Filipiak et al. (2009). These conditions are identical to the uniqueness condition of the least squares estimator of $\boldsymbol{\Xi}$. 
Let us consider Models I and II. We are interested in estimation of linear functions of $\mathbf{B}_{i}, i=1,2,3$, which can be presented as $\mathbf{K B}_{i} \mathbf{L}$. The cases $i=2,3$ have been presented in Filipiak et al. (2009). Thus, we will prove only case (i) of the theorem below.

The bearing idea of the proof is the following. If $\Sigma$ is known we have a usual Gauss-Markov model. In this case all estimators satisfy, for given $\Sigma$, (4), (5) and (6). However, it will appear that the uniqueness conditions depend only on the design matrices $\mathbf{A}_{i}$ and $\mathbf{C}_{i}$ and are completely unrelated with $\boldsymbol{\Sigma}$. Thus, for all values of $\boldsymbol{\Sigma}$, including the MLE, the same conditions for uniqueness are obtained. Hence, we have the complete solution to uniqueness/estimation problems for the EGCM and it suffices to consider models with known $\boldsymbol{\Sigma}$. Moreover, it is noted that we immediately obtain conditions for both Model I and Model II and $\widehat{\boldsymbol{\Sigma}}$ in both models is always uniquely estimated.

Theorem 3 The linear functions $\mathbf{K B}_{i} \mathbf{L}, i=1,2,3$, are estimable in Model I if and only if

(i) $\mathcal{R}\left(\mathbf{L} \otimes \mathbf{K}^{\prime}\right) \subseteq \mathcal{R}\left(\mathbf{C}_{1} \mathbf{Q}_{C_{2}^{\prime}} \otimes \mathbf{A}_{1}^{\prime}\right)+\mathcal{R}\left(\mathbf{C}_{1} \mathbf{P}_{C_{2}^{\prime}} \mathbf{Q}_{C_{3}^{\prime}} \otimes \mathbf{A}_{1}^{\prime} \mathbf{Q}_{A_{2}}\right)$

$$
+\mathcal{R}\left(\mathbf{C}_{1} \mathbf{P}_{C_{3}^{\prime}} \otimes \mathbf{A}_{1}^{\prime} \mathbf{Q}_{\left(A_{2}: A_{3}\right)}\right), \quad \text { for } i=1,
$$

(ii) $\mathcal{R}\left(\mathbf{L} \otimes \mathbf{K}^{\prime}\right) \subseteq \mathcal{R}\left(\mathbf{C}_{2} \mathbf{Q}_{C_{3}^{\prime}} \otimes \mathbf{A}_{2}^{\prime} \mathbf{Q}_{A_{1}}\right)+\mathcal{R}\left(\mathbf{C}_{2} \mathbf{P}_{C_{3}^{\prime}} \otimes \mathbf{A}_{2}^{\prime} \mathbf{Q}_{\left(A_{1}: A_{3}\right)}\right)$, for $i=2$,

(iii) $\mathcal{R}\left(\mathbf{L} \otimes \mathbf{K}^{\prime}\right) \subseteq \mathcal{R}\left(\mathbf{C}_{3} \otimes \mathbf{A}_{3}^{\prime} \mathbf{Q}_{\left(A_{1}: A_{2}\right)}\right), \quad$ for $i=3$.

Proof Let consider Model I and let $i=1$. Using the "vec" operator and by elimination of nuisance parameters (first $\mathbf{B}_{2}$ and then $\mathbf{B}_{3}$ ) we obtain the estimability condition

$$
\mathcal{R}\left(\mathbf{L} \otimes \mathbf{K}^{\prime}\right) \subseteq \mathcal{R}\left(\left(\mathbf{C}_{1} \otimes \mathbf{A}_{1}^{\prime}\right) \mathbf{Q}_{C_{2}^{\prime} \otimes A_{2}} \mathbf{Q}_{Q_{C_{2}^{\prime} \otimes A_{2}}\left(C_{3}^{\prime} \otimes A_{3}\right)}\right)
$$

Since $\mathcal{R}\left(\mathbf{C}_{3}^{\prime}\right) \subseteq \mathcal{R}\left(\mathbf{C}_{2}^{\prime}\right) \subseteq \mathcal{R}\left(\mathbf{C}_{1}^{\prime}\right)$ we have

$$
\left(\mathbf{C}_{1} \otimes \mathbf{A}_{1}^{\prime}\right) \mathbf{Q}_{C_{2}^{\prime} \otimes A_{2}}=\mathbf{C}_{1} \mathbf{Q}_{C_{2}^{\prime}} \otimes \mathbf{A}_{1}^{\prime}+\mathbf{C}_{1} \mathbf{P}_{C_{2}^{\prime}} \otimes \mathbf{A}_{1}^{\prime} \mathbf{Q}_{A_{2}}
$$

and

$$
\mathbf{Q}_{C_{2}^{\prime} \otimes A_{2}}\left(\mathbf{C}_{3}^{\prime} \otimes \mathbf{A}_{3}\right)=\mathbf{C}_{3}^{\prime} \otimes \mathbf{Q}_{A_{2}} \mathbf{A}_{3}
$$

Thus

$$
\begin{aligned}
\left(\mathbf{C}_{1} \otimes \mathbf{A}_{1}^{\prime}\right) \mathbf{Q}_{C_{2}^{\prime} \otimes A_{2}} \mathbf{Q}_{Q_{C_{2}^{\prime} \otimes A_{2}}\left(C_{3}^{\prime} \otimes A_{3}\right)} & \\
& =\left(\mathbf{C}_{1} \mathbf{Q}_{C_{2}^{\prime}} \otimes \mathbf{A}_{1}^{\prime}+\mathbf{C}_{1} \mathbf{P}_{C_{2}^{\prime}} \otimes \mathbf{A}_{1}^{\prime} \mathbf{Q}_{A_{2}}\right) \mathbf{Q}_{C_{3}^{\prime} \otimes Q_{A_{2}} A_{3}} .
\end{aligned}
$$


Using the fact that $\mathbf{P}_{C_{2}^{\prime}} \mathbf{P}_{C_{3}^{\prime}}=\mathbf{P}_{C_{3}^{\prime}}$ we have $\mathbf{Q}_{C_{2}^{\prime}} \mathbf{P}_{C_{3}^{\prime}}=\mathbf{0}$, and from the idempotent property of $\mathbf{Q}_{A_{2}}$ and the property $\mathbf{P}_{Q_{A_{2}} A_{3}}=\mathbf{P}_{\left(A_{2}: A_{3}\right)}-\mathbf{P}_{A_{2}}$ we get

$$
\begin{aligned}
& \left(\mathbf{C}_{1} \mathbf{Q}_{C_{2}^{\prime}} \otimes \mathbf{A}_{1}^{\prime}+\mathbf{C}_{1} \mathbf{P}_{C_{2}^{\prime}} \otimes \mathbf{A}_{1}^{\prime} \mathbf{Q}_{A_{2}}\right) \mathbf{Q}_{C_{3}^{\prime} \otimes Q_{A_{2}} A_{3}} \\
& \quad=\mathbf{C}_{1} \mathbf{Q}_{C_{2}^{\prime}} \otimes \mathbf{A}_{1}^{\prime}+\mathbf{C}_{1} \mathbf{P}_{C_{2}^{\prime}} \mathbf{Q}_{C_{3}^{\prime}} \otimes \mathbf{A}_{1}^{\prime} \mathbf{Q}_{A_{2}}+\mathbf{C}_{1} \mathbf{P}_{C_{3}^{\prime}} \otimes \mathbf{A}_{1}^{\prime} \mathbf{Q}_{\left(A_{2}: A_{3}\right)} .
\end{aligned}
$$

Since $\mathcal{R}(\mathbf{X})=\mathcal{R}\left(\mathbf{X X}^{\prime}\right)$ we obtain

$$
\begin{aligned}
\mathcal{R}\left(\mathbf{L} \otimes \mathbf{K}^{\prime}\right) \subseteq \mathcal{R}( & \left(\mathbf{C}_{1} \mathbf{Q}_{C_{2}^{\prime}} \mathbf{C}_{1}^{\prime} \otimes \mathbf{A}_{1}^{\prime} \mathbf{A}_{1}+\mathbf{C}_{1} \mathbf{P}_{C_{2}^{\prime}} \mathbf{Q}_{C_{3}^{\prime}} \mathbf{P}_{C_{2}^{\prime}} \mathbf{C}_{1}^{\prime} \otimes \mathbf{A}_{1}^{\prime} \mathbf{Q}_{A_{2}} \mathbf{A}_{1}\right. \\
& \left.+\mathbf{C}_{1} \mathbf{P}_{C_{3}^{\prime}} \mathbf{C}_{1}^{\prime} \otimes \mathbf{A}_{1}^{\prime} \mathbf{Q}_{\left(A_{2}: A_{3}\right)} \mathbf{A}_{1}\right)
\end{aligned}
$$

and the nonnegative definiteness of the components in the sum implies (i).

Theorem 4 The linear functions $\mathbf{K B}_{i} \mathbf{L}, i=1,2,3$, are estimable in Model II if and only if

(i) $\mathcal{R}\left(\mathbf{L} \otimes \mathbf{K}^{\prime}\right) \subseteq \mathcal{R}\left(\mathbf{C}_{1} \otimes \mathbf{A}_{1}^{\prime} \mathbf{Q}_{A_{2}}\right)+\mathcal{R}\left(\mathbf{C}_{1} \mathbf{Q}_{C_{2}^{\prime}} \otimes \mathbf{A}_{1}^{\prime} \mathbf{P}_{A_{2}} \mathbf{Q}_{A_{3}}\right)$

$$
+\mathcal{R}\left(\mathbf{C}_{1} \mathbf{Q}_{\left(C_{2}^{\prime}: C_{3}^{\prime}\right)} \otimes \mathbf{A}_{1}^{\prime} \mathbf{P}_{A_{3}}\right), \quad \text { for } i=1,
$$

(ii) $\mathcal{R}\left(\mathbf{L} \otimes \mathbf{K}^{\prime}\right) \subseteq \mathcal{R}\left(\mathbf{C}_{2} \mathbf{Q}_{C_{1}^{\prime}} \otimes \mathbf{A}_{2}^{\prime} \mathbf{Q}_{A_{3}}\right)+\mathcal{R}\left(\mathbf{C}_{2} \mathbf{Q}_{\left(C_{1}^{\prime}: C_{3}^{\prime}\right)} \otimes \mathbf{A}_{2}^{\prime} \mathbf{P}_{A_{3}}\right)$, for $i=2$,

(iii) $\mathcal{R}\left(\mathbf{L} \otimes \mathbf{K}^{\prime}\right) \subseteq \mathcal{R}\left(\mathbf{C}_{3} \mathbf{Q}_{\left(C_{1}^{\prime}: C_{2}^{\prime}\right)} \otimes \mathbf{A}_{3}^{\prime}\right), \quad$ for $i=3$.

Proof Replacing $\mathcal{R}\left(\mathbf{C}_{3}^{\prime}\right) \subseteq \mathcal{R}\left(\mathbf{C}_{2}^{\prime}\right) \subseteq \mathcal{R}\left(\mathbf{C}_{1}^{\prime}\right)$ by $\mathcal{R}\left(\mathbf{A}_{3}\right) \subseteq \mathcal{R}\left(\mathbf{A}_{2}\right) \subseteq \mathcal{R}\left(\mathbf{A}_{1}\right)$, the proof follows similarly to the previous one.

It is worth noting, that the column spaces in both theorems are not necessarily disjoint (see e.g. Case 2 in the Example 1). Thus, respective sums cannot be replaced by orthogonal sums.

The next corollary is an immediate consequence of the theorems.

Corollary 1 Under Model I (Model II) with known $\mathbf{\Sigma}$, the least squares estimator of a linear function of $\mathbf{B}_{i}, i=1,2,3$, is unique if and only if the conditions of Theorem 3 (Theorem 4) are satisfied.

In order to guarantee uniqueness of $\widehat{\mathbf{B}}_{i}, i=1,2,3$, it is necessary to cancel in Theorem 1 (Theorem 2) the expressions which involve $\mathbf{Z}_{k l}, k, l=1,2$, 3. For Model I the immediate consequence of this fact is the following corollary.

\section{Corollary 2 In Model I}

(i) $\widehat{\mathbf{B}}_{1}$ is unique if and only if

$$
\begin{aligned}
& \operatorname{rank}\left(\mathbf{A}_{1}\right)=m_{1}, \quad \operatorname{rank}\left(\mathbf{C}_{1}\right)=q_{1}, \quad \mathcal{R}\left(\mathbf{A}_{1}\right) \cap \mathcal{R}\left(\mathbf{A}_{2}\right)=\{\mathbf{0}\}, \\
& \mathcal{R}\left(\mathbf{A}_{2}\right)^{\perp} \cap \mathcal{R}\left(\mathbf{A}_{1}: \mathbf{A}_{2}\right) \cap \mathcal{R}\left(\mathbf{A}_{2}: \mathbf{A}_{3}\right)=\{\mathbf{0}\} ;
\end{aligned}
$$


(ii) $\widehat{\mathbf{B}}_{2}$ is unique if and only if

$$
\begin{aligned}
& \operatorname{rank}\left(\mathbf{A}_{2}\right)=m_{2}, \quad \operatorname{rank}\left(\mathbf{C}_{2}\right)=q_{2}, \quad \mathcal{R}\left(\mathbf{A}_{1}\right) \cap \mathcal{R}\left(\mathbf{A}_{2}\right)=\{\mathbf{0}\}, \\
& \mathcal{R}\left(\mathbf{A}_{1}\right)^{\perp} \cap \mathcal{R}\left(\mathbf{A}_{1}: \mathbf{A}_{2}\right) \cap \mathcal{R}\left(\mathbf{A}_{1}: \mathbf{A}_{3}\right)=\{\mathbf{0}\}
\end{aligned}
$$

(iii) $\widehat{\mathbf{B}}_{3}$ is unique if and only if

$$
\operatorname{rank}\left(\mathbf{A}_{3}\right)=m_{3}, \quad \operatorname{rank}\left(\mathbf{C}_{3}\right)=q_{3}, \quad \mathcal{R}\left(\mathbf{A}_{3}\right) \cap \mathcal{R}\left(\mathbf{A}_{1}: \mathbf{A}_{2}\right)=\{\mathbf{0}\} .
$$

For Model II conditions for uniqueness of estimators are given in Kollo and von Rosen (2005) [Theorem 4.1.12, p. 396].

\section{Moments}

Since the distributions of the MLEs are difficult to derive one has to focus on moments. They can be used to validate the model as well as be used in density approximations, e.g. Edgeworth expansions. Before considering dispersion matrices of the MLEs of the mean parameters we note that they are unbiased.

Theorem 5 Suppose that in Model I the MLEs $\mathbf{K} \widehat{\mathbf{B}}_{i} \mathbf{L}, i=1,2,3$, are uniquely estimated. Then $\mathbf{K} \widehat{\mathbf{B}}_{i} \mathbf{L}, i=1,2,3$, are unbiased estimators of $\mathbf{K B}_{i} \mathbf{L}, i=1,2,3$.

Proof $\mathbf{K} \widehat{\mathbf{B}}_{3} \mathbf{L}$ is unbiased since $\mathbf{S}_{1}$ is independent of $\mathbf{A}_{3}^{\prime} \mathbf{Q}_{\left(A_{1}: A_{2}\right)} \mathbf{Y}$.

$\mathbf{K} \widehat{\mathbf{B}}_{2} \mathbf{L}$ is unbiased since $\mathbf{S}_{2}$ is independent of $\mathbf{A}_{2}^{\prime} \mathbf{Q}_{A_{1}} \mathbf{Y}$ and $\mathcal{R}\left(\mathbf{C}_{3}^{\prime}\right) \subseteq \mathcal{R}\left(\mathbf{C}_{2}^{\prime}\right)$.

$\mathbf{K} \widehat{\mathbf{B}}_{1} \mathbf{L}$ is unbiased since $\mathbf{S}_{3}$ is independent of $\mathbf{A}_{1}^{\prime} \mathbf{Y}$ and $\mathcal{R}\left(\mathbf{C}_{3}^{\prime}\right) \subseteq \mathcal{R}\left(\mathbf{C}_{2}^{\prime}\right) \subseteq \mathcal{R}\left(\mathbf{C}_{1}^{\prime}\right)$.

For Model II the corresponding result is given in Kollo and von Rosen (2005) [Theorem 4.2.6, p. 430].

Theorem 6 Suppose that in Model I the MLEs $\mathbf{K} \widehat{\mathbf{B}}_{i} \mathbf{L}, i=1,2,3$, are uniquely estimated. Let

$$
\begin{aligned}
\gamma_{1} & =\frac{n-\operatorname{rank}\left(A_{1}: A_{2}: A_{3}\right)-1}{n-\operatorname{rank}\left(A_{1}: A_{2}: A_{3}\right)-p+\operatorname{rank}\left(C_{3}\right)-1}, \\
\gamma_{2}= & \frac{p-\operatorname{rank}\left(C_{2}\right)}{n-\operatorname{rank}\left(A_{1}: A_{2}\right)-p+\operatorname{rank}\left(C_{2}\right)-1}, \\
\gamma_{3}= & \frac{\left(n-\operatorname{rank}\left(A_{1}: A_{2}\right)-p+\operatorname{rank}\left(C_{3}\right)-1\right)\left(p-\operatorname{rank}\left(C_{2}\right)\right)}{\left(n-\operatorname{rank}\left(A_{1}: A_{2}: A_{3}\right)-p+\operatorname{rank}\left(C_{3}\right)-1\right)\left(n-\operatorname{rank}\left(A_{1}: A_{2}\right)-p+\operatorname{rank}\left(C_{2}\right)-1\right)}, \\
\gamma_{4}= & \frac{p-\operatorname{rank}\left(C_{1}\right)}{n-\operatorname{rank}\left(A_{1}\right)-p+\operatorname{rank}\left(C_{1}\right)-1}, \quad \gamma_{5}=\frac{n-\operatorname{rank}\left(A_{1}: A_{2}\right)-p+\operatorname{rank}\left(C_{3}\right)-1}{n-\operatorname{rank}\left(A_{1}: A_{2}: A_{3}\right)-p+\operatorname{rank}\left(C_{3}\right)-1}, \\
\gamma_{6}= & \frac{n-\operatorname{rank}\left(A_{1}\right)-p+\operatorname{rank}\left(C_{2}\right)-1}{n-\operatorname{rank}\left(A_{1}: A_{2}\right)-p+\operatorname{rank}\left(C_{2}\right)-1}, \quad \gamma_{7}=\frac{n-\operatorname{rank}\left(A_{1}: A_{2}\right)}{n-\operatorname{rank}\left(A_{1}\right)}, \\
\gamma_{8}= & \frac{n-\operatorname{rank}\left(A_{1}\right)-p+\operatorname{rank}\left(C_{1}\right)-1}{n-\operatorname{rank}\left(A_{1}: A_{2}\right)-p+\operatorname{rank}\left(C_{1}\right)-1} .
\end{aligned}
$$

Then, 
(i) If $\gamma_{1}>0$

$$
D\left[\mathbf{K} \widehat{\mathbf{B}}_{3} \mathbf{L}\right]=\gamma_{1} \mathbf{L}^{\prime}\left(\mathbf{C}_{3} \boldsymbol{\Sigma}^{-1} \mathbf{C}_{3}^{\prime}\right)^{-} \mathbf{L} \otimes \mathbf{K}\left(\mathbf{A}_{3}^{\prime} \mathbf{Q}_{\left(A_{1}: A_{2}\right)} \mathbf{A}_{3}\right)^{-} \mathbf{K}^{\prime} .
$$

(ii) If $\gamma_{1}>0, \gamma_{2}>0$ and $\gamma_{3}>0$

$$
\begin{aligned}
D\left[\mathbf{K} \widehat{\mathbf{B}}_{2} \mathbf{L}\right]= & \gamma_{1} \mathbf{L}^{\prime} \boldsymbol{\Sigma}^{1 / 2} \mathbf{P}_{\Sigma^{-1 / 2} C_{3}^{\prime}} \boldsymbol{\Sigma}^{1 / 2} \mathbf{L} \otimes \mathbf{K}\left(\mathbf{A}_{2}^{\prime} \mathbf{Q}_{\left(A_{1}: A_{3}\right)} \mathbf{A}_{2}\right)^{-} \mathbf{K}^{\prime} \\
& +\mathbf{L}^{\prime} \boldsymbol{\Sigma}^{1 / 2}\left[\left(1+\gamma_{2}\right) \mathbf{P}_{\Sigma^{-1 / 2} C_{2}^{\prime}}+\left(\gamma_{3}-\gamma_{2}-\gamma_{1}\right) \mathbf{P}_{\Sigma^{-1 / 2} C_{3}^{\prime}}\right] \boldsymbol{\Sigma}^{1 / 2} \mathbf{L} \\
& \otimes \mathbf{K}\left(\mathbf{A}_{2}^{\prime} \mathbf{Q}_{A_{1}} \mathbf{A}_{2}\right)^{-} \mathbf{K}^{\prime} .
\end{aligned}
$$

(iii) $i$ If $\gamma_{i}>0, i=1, \ldots, 8$,

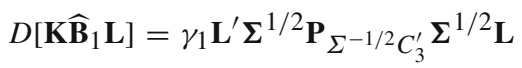

$$
\begin{aligned}
& \otimes \mathbf{K}\left(\mathbf{A}_{1}^{\prime} \mathbf{A}_{1}\right)^{-} \mathbf{A}_{1}^{\prime}\left(\mathbf{I}-\mathbf{A}_{2}\left(\mathbf{A}_{2}^{\prime} \mathbf{Q}_{A_{1}} \mathbf{A}_{2}\right)^{-} \mathbf{A}_{2}^{\prime} \mathbf{Q}_{A_{1}}\right) \mathbf{A}_{3}\left(\mathbf{A}_{3}^{\prime} \mathbf{Q}_{\left(A_{1}: A_{2}\right)} \mathbf{A}_{3}\right)^{-} \mathbf{A}_{3}^{\prime} \\
& \times\left(\mathbf{I}-\mathbf{Q}_{A_{1}} \mathbf{A}_{2}\left(\mathbf{A}_{2}^{\prime} \mathbf{Q}_{A_{1}} \mathbf{A}_{2}\right)^{-} \mathbf{A}_{2}^{\prime}\right) \mathbf{A}_{1}\left(\mathbf{A}_{1}^{\prime} \mathbf{A}_{1}\right)^{-} \mathbf{K}^{\prime} \\
& +\mathbf{L}^{\prime} \boldsymbol{\Sigma}^{1 / 2}\left[\left(1+\gamma_{2}\right) \mathbf{P}_{\Sigma^{-1 / 2} C_{2}^{\prime}}+\left(\gamma_{3}-\gamma_{2}\right) \mathbf{P}_{\Sigma^{-1 / 2} C_{3}^{\prime}}\right] \boldsymbol{\Sigma}^{1 / 2} \mathbf{L} \\
& \otimes \mathbf{K}\left(\mathbf{A}_{1}^{\prime} \mathbf{Q}_{A_{2}} \mathbf{A}_{1}\right)^{-} \mathbf{K}^{\prime} \\
& +\mathbf{L}^{\prime} \boldsymbol{\Sigma}^{1 / 2}\left[\left(1+\gamma_{4}\right) \mathbf{P}_{\Sigma^{-1 / 2} C_{1}^{\prime}}-\left(1+\gamma_{2}+\gamma_{4}\right) \mathbf{P}_{\Sigma^{-1 / 2} C_{2}^{\prime}}\right. \\
& \left.+\left(\gamma_{4} \gamma_{5} \gamma_{6}+\gamma_{2}-\gamma_{3}\right) \mathbf{P}_{\Sigma^{-1 / 2} C_{3}^{\prime}}+\gamma_{4} \gamma_{6} \mathbf{P}_{Q_{\Sigma^{-1 / 2} C_{3}^{\prime}} \Sigma^{-1 / 2} C_{2}^{\prime}}\right] \boldsymbol{\Sigma}^{1 / 2} \mathbf{L} \\
& \otimes \mathbf{K}\left(\mathbf{A}_{1}^{\prime} \mathbf{A}_{1}\right)^{-} \mathbf{K}^{\prime} .
\end{aligned}
$$

Proof The proofs of (i) and (ii) are given below whereas the proof of (iii), because of lengthy calculations and similarities with (ii), is presented in the Appendix B.

Proof of (i): First observe that

$$
\mathbf{K}\left(\widehat{\mathbf{B}}_{3}-\mathbf{B}_{3}\right) \mathbf{L}=\mathbf{K}\left(\mathbf{A}_{3}^{\prime} \mathbf{Q}_{\left(A_{1}: A_{2}\right)} \mathbf{A}_{3}\right)^{-} \mathbf{A}_{3}^{\prime} \mathbf{Q}_{\left(A_{1}: A_{2}\right)}(\mathbf{Y}-E[\mathbf{Y}]) \mathbf{S}_{1}^{-1} \mathbf{C}_{3}^{\prime}\left(\mathbf{C}_{3} \mathbf{S}_{1}^{-1} \mathbf{C}_{3}^{\prime}\right)^{-} \mathbf{L}
$$

Since $\mathbf{A}_{3}^{\prime} \mathbf{Q}_{\left(A_{1}: A_{2}\right)} \mathbf{Y}$ is independent of $\mathbf{S}_{1}$

$$
\begin{gathered}
D\left[\mathbf{K} \widehat{\mathbf{B}}_{3} \mathbf{L}\right]=E\left[\mathbf{L}^{\prime}\left(\mathbf{C}_{3} \mathbf{S}_{1}^{-1} \mathbf{C}_{3}^{\prime}\right)^{-} \mathbf{C}_{3} \mathbf{S}_{1}^{-1} \boldsymbol{\Sigma} \mathbf{S}_{1}^{-1} \mathbf{C}_{3}^{\prime}\left(\mathbf{C}_{3} \mathbf{S}_{1}^{-1} \mathbf{C}_{3}^{\prime}\right)^{-} \mathbf{L}\right] \\
\otimes \mathbf{K}\left(\mathbf{A}_{3}^{\prime} \mathbf{Q}_{\left(A_{1}: A_{2}\right)} \mathbf{A}_{3}\right)^{-} \mathbf{K}^{\prime} .
\end{gathered}
$$

However, $\mathbf{S}_{1} \sim W_{p}\left(\boldsymbol{\Sigma}, n-\operatorname{rank}\left(\mathbf{A}_{1}: \mathbf{A}_{2}: \mathbf{A}_{3}\right)\right)$ and via some calculations, see Kollo and von Rosen (2005) [(4.2.18)-(4.2.23), p. 413], the statement follows. 
Proof of (ii): From Theorem 1 it follows that

$$
\begin{aligned}
D\left[\mathbf{K} \widehat{\mathbf{B}}_{2} \mathbf{L}\right]= & D\left[\mathbf{K}\left(\mathbf{A}_{2}^{\prime} \mathbf{Q}_{A_{1}} \mathbf{A}_{2}\right)^{-} \mathbf{A}_{2}^{\prime} \mathbf{Q}_{A_{1}}(\mathbf{Y}-E[\mathbf{Y}]) \mathbf{S}_{2}^{-1} \mathbf{C}_{2}^{\prime}\left(\mathbf{C}_{2} \mathbf{S}_{2}^{-1} \mathbf{C}_{2}^{\prime}\right)^{-} \mathbf{L}\right] \\
& +D\left[\mathbf{K}\left(\mathbf{A}_{2}^{\prime} \mathbf{Q}_{A_{1}} \mathbf{A}_{2}\right)^{-} \mathbf{A}_{2}^{\prime} \mathbf{Q}_{A_{1}} \mathbf{A}_{3}\left(\widehat{\mathbf{B}}_{3}-\mathbf{B}_{3}\right) \mathbf{C}_{3} \mathbf{S}_{2}^{-1} \mathbf{C}_{2}^{\prime}\left(\mathbf{C}_{2} \mathbf{S}_{2}^{-1} \mathbf{C}_{2}^{\prime}\right)^{-} \mathbf{L}\right] \\
& +\operatorname{Cov}\left[\mathbf{K}\left(\mathbf{A}_{2}^{\prime} \mathbf{Q}_{A_{1}} \mathbf{A}_{2}\right)^{-} \mathbf{A}_{2}^{\prime} \mathbf{Q}_{A_{1}}(\mathbf{Y}-E[\mathbf{Y}]) \mathbf{S}_{2}^{-1} \mathbf{C}_{2}^{\prime}\left(\mathbf{C}_{2} \mathbf{S}_{2}^{-1} \mathbf{C}_{2}^{\prime}\right)^{-} \mathbf{L},\right. \\
& \left.\mathbf{K}\left(\mathbf{A}_{2}^{\prime} \mathbf{Q}_{A_{1}} \mathbf{A}_{2}\right)^{-} \mathbf{A}_{2}^{\prime} \mathbf{Q}_{A_{1}} \mathbf{A}_{3}\left(\widehat{\mathbf{B}}_{3}-\mathbf{B}_{3}\right) \mathbf{C}_{3} \mathbf{S}_{2}^{-1} \mathbf{C}_{2}^{\prime}\left(\mathbf{C}_{2} \mathbf{S}_{2}^{-1} \mathbf{C}_{2}^{\prime}\right)^{-} \mathbf{L}\right] \\
& +\operatorname{Cov}\left[\mathbf{K}\left(\mathbf{A}_{2}^{\prime} \mathbf{Q}_{A_{1}} \mathbf{A}_{2}\right)^{-} \mathbf{A}_{2}^{\prime} \mathbf{Q}_{A_{1}} \mathbf{A}_{3}\left(\widehat{\mathbf{B}}_{3}-\mathbf{B}_{3}\right) \mathbf{C}_{3} \mathbf{S}_{2}^{-1} \mathbf{C}_{2}^{\prime}\left(\mathbf{C}_{2} \mathbf{S}_{2}^{-1} \mathbf{C}_{2}^{\prime}\right)^{-} \mathbf{L},\right. \\
& \left.\mathbf{K}\left(\mathbf{A}_{2}^{\prime} \mathbf{Q}_{A_{1}} \mathbf{A}_{2}\right)^{-} \mathbf{A}_{2}^{\prime} \mathbf{Q}_{A_{1}}(\mathbf{Y}-E[\mathbf{Y}]) \mathbf{S}_{2}^{-1} \mathbf{C}_{2}^{\prime}\left(\mathbf{C}_{2} \mathbf{S}_{2}^{-1} \mathbf{C}_{2}^{\prime}\right)^{-} \mathbf{L}\right] .
\end{aligned}
$$

Because of independence between $\mathbf{A}_{2}^{\prime} \mathbf{Q}_{A_{1}} \mathbf{Y}$ and $\mathbf{S}_{1}$ and between $\mathbf{A}_{2}^{\prime} \mathbf{Q}_{A_{1}} \mathbf{Y}$ and $\mathbf{Q}_{\left(A_{1}: A_{2}\right)} \mathbf{Y}$, because of unbiasedness of $\mathbf{B}_{3}$, and since $\mathcal{R}\left(\mathbf{C}_{3}^{\prime}\right) \subseteq \mathcal{R}\left(\mathbf{C}_{2}^{\prime}\right)$ implies

$$
\mathbf{C}_{3} \mathbf{S}_{2}^{-1} \mathbf{C}_{2}^{\prime}\left(\mathbf{C}_{2} \mathbf{S}_{2}^{-1} \mathbf{C}_{2}^{\prime}\right)^{-} \mathbf{C}_{2}=\mathbf{C}_{3}
$$

we obtain

$$
\begin{gathered}
\operatorname{Cov}\left[\mathbf{K}\left(\mathbf{A}_{2}^{\prime} \mathbf{Q}_{A_{1}} \mathbf{A}_{2}\right)^{-} \mathbf{A}_{2}^{\prime} \mathbf{Q}_{A_{1}}(\mathbf{Y}-E[\mathbf{Y}]) \mathbf{S}_{2}^{-1} \mathbf{C}_{2}^{\prime}\left(\mathbf{C}_{2} \mathbf{S}_{2}^{-1} \mathbf{C}_{2}^{\prime}\right)^{-} \mathbf{L},\right. \\
\left.\mathbf{K}\left(\mathbf{A}_{2}^{\prime} \mathbf{Q}_{A_{1}} \mathbf{A}_{2}\right)^{-} \mathbf{A}_{2}^{\prime} \mathbf{Q}_{A_{1}} \mathbf{A}_{3}\left(\widehat{\mathbf{B}}_{3}-\mathbf{B}_{3}\right) \mathbf{C}_{3} \mathbf{S}_{2}^{-1} \mathbf{C}_{2}^{\prime}\left(\mathbf{C}_{2} \mathbf{S}_{2}^{-1} \mathbf{C}_{2}^{\prime}\right)^{-} \mathbf{L}\right] \\
=\operatorname{Cov}\left[\mathbf{K}\left(\mathbf{A}_{2}^{\prime} \mathbf{Q}_{A_{1}} \mathbf{A}_{2}\right)^{-} \mathbf{A}_{2}^{\prime} \mathbf{Q}_{A_{1}}(\mathbf{Y}-E[\mathbf{Y}]) \mathbf{C}_{2}^{\prime}\left(\mathbf{C}_{2} \mathbf{C}_{2}^{\prime}\right)^{-} \mathbf{L},\right. \\
\left.\mathbf{K}\left(\mathbf{A}_{2}^{\prime} \mathbf{Q}_{A_{1}} \mathbf{A}_{2}\right)^{-} \mathbf{A}_{2}^{\prime} \mathbf{Q}_{A_{1}} \mathbf{A}_{3}\left(\widehat{B}_{3}-\mathbf{B}_{3}\right) \mathbf{C}_{3} \mathbf{C}_{2}^{\prime}\left(\mathbf{C}_{2} \mathbf{C}_{2}^{\prime}\right)^{-} \mathbf{L}\right]=\mathbf{0} .
\end{gathered}
$$

Thus, $D\left[\mathbf{K} \widehat{\mathbf{B}}_{2} \mathbf{L}\right]$ equals the sum of (7) and (8) and we are going to consider these terms separately. However, we immediately obtain from (i) that (8) equals

$$
\begin{aligned}
D[ & \left.\mathbf{K}\left(\mathbf{A}_{2}^{\prime} \mathbf{Q}_{A_{1}} \mathbf{A}_{2}\right)^{-} \mathbf{A}_{2}^{\prime} \mathbf{Q}_{A_{1}} \mathbf{A}_{3}\left(\widehat{\mathbf{B}}_{3}-\mathbf{B}_{3}\right) \mathbf{C}_{3} \mathbf{S}_{2}^{-1} \mathbf{C}_{2}^{\prime}\left(\mathbf{C}_{2} \mathbf{S}_{2}^{-1} \mathbf{C}_{2}^{\prime}\right)^{-} \mathbf{L}\right] \\
= & \gamma_{1} \mathbf{L}^{\prime} \mathbf{C}_{3}^{\prime}\left(\mathbf{C}_{3} \boldsymbol{\Sigma}^{-1} \mathbf{C}_{3}^{\prime}\right)^{-} \mathbf{C}_{3} \mathbf{L} \otimes \mathbf{K}\left(\mathbf{A}_{2}^{\prime} \mathbf{Q}_{A_{1}} \mathbf{A}_{2}\right)^{-} \\
& \times \mathbf{A}_{2}^{\prime} \mathbf{Q}_{A_{1}} \mathbf{A}_{3}\left(\mathbf{A}_{3}^{\prime} \mathbf{Q}_{\left(A_{1}: A_{2}\right)} \mathbf{A}_{3}\right)^{-} \mathbf{A}_{3}^{\prime} \mathbf{Q}_{A_{1}} \mathbf{A}_{2}\left(\mathbf{A}_{2}^{\prime} \mathbf{Q}_{A_{1}} \mathbf{A}_{2}\right)^{-} \mathbf{K}^{\prime} .
\end{aligned}
$$

Observe, that since $\mathbf{Q}_{\left(A_{1}: A_{2}\right)}$ and $\mathbf{Q}_{A_{1}}$ are nonnegative definite, using the formula for the generalized inverse of a partitioned matrix from Kollo and von Rosen (2005) [Proposition 1.3.7, p. 76], we obtain

$$
\begin{aligned}
& \left(\mathbf{A}_{2}^{\prime} \mathbf{Q}_{A_{1}} \mathbf{A}_{2}\right)^{-} \mathbf{A}_{2}^{\prime} \mathbf{Q}_{A_{1}} \mathbf{A}_{3}\left(\mathbf{A}_{3}^{\prime} \mathbf{Q}_{\left(A_{1}: A_{2}\right)} \mathbf{A}_{3}\right)^{-} \mathbf{A}_{3}^{\prime} \mathbf{Q}_{A_{1}} \mathbf{A}_{2}\left(\mathbf{A}_{2}^{\prime} \mathbf{Q}_{A_{1}} \mathbf{A}_{2}\right)^{-} \\
& \quad=\left(\mathbf{A}_{2}^{\prime} \mathbf{Q}_{\left(A_{1}: A_{3}\right)} \mathbf{A}_{2}\right)^{-}-\left(\mathbf{A}_{2}^{\prime} \mathbf{Q}_{A_{1}} \mathbf{A}_{2}\right)^{-}
\end{aligned}
$$

and

$$
\begin{aligned}
& D\left[\mathbf{K}\left(\mathbf{A}_{2}^{\prime} \mathbf{Q}_{A_{1}} \mathbf{A}_{2}\right)^{-} \mathbf{A}_{2}^{\prime} \mathbf{Q}_{A_{1}} \mathbf{A}_{3}\left(\widehat{\mathbf{B}}_{3}-\mathbf{B}_{3}\right) \mathbf{C}_{3} \mathbf{S}_{2}^{-1} \mathbf{C}_{2}^{\prime}\left(\mathbf{C}_{2} \mathbf{S}_{2}^{-1} \mathbf{C}_{2}^{\prime}\right)^{-} \mathbf{L}\right] \\
& \quad=\gamma_{1} \mathbf{L}^{\prime} \boldsymbol{\Sigma}^{1 / 2} \mathbf{P}_{\Sigma^{-1 / 1} C_{3}^{\prime}} \boldsymbol{\Sigma}^{1 / 2} \mathbf{L} \otimes \mathbf{K}\left[\left(\mathbf{A}_{2}^{\prime} \mathbf{Q}_{\left(A_{1}: A_{3}\right)} \mathbf{A}_{2}\right)^{-}-\left(\mathbf{A}_{2}^{\prime} \mathbf{Q}_{A_{1}} \mathbf{A}_{2}\right)^{-}\right] \mathbf{K}^{\prime} .
\end{aligned}
$$


Now (7) is exploited. The independence between $\mathbf{A}_{2}^{\prime} \mathbf{Q}_{A_{1}} \mathbf{Y}$ and $\mathbf{S}_{2}$ yields that (7) equals

$$
E\left[\mathbf{L}^{\prime}\left(\mathbf{C}_{2} \mathbf{S}_{2}^{-1} \mathbf{C}_{2}^{\prime}\right)^{-} \mathbf{C}_{2} \mathbf{S}_{2}^{-1} \boldsymbol{\Sigma} \mathbf{S}_{2}^{-1} \mathbf{C}_{2}^{\prime}\left(\mathbf{C}_{2} \mathbf{S}_{2}^{-1} \mathbf{C}_{2}^{\prime}\right)^{-} \mathbf{L}\right] \otimes \mathbf{K}\left(\mathbf{A}_{2}^{\prime} \mathbf{Q}_{A_{1}} \mathbf{A}_{2}\right)^{-} \mathbf{K}^{\prime}
$$

The expectation in (10) will be considered in detail and

$$
\mathbf{F}_{1}=E\left[\mathbf{P}_{C_{2}^{\prime} ; S_{2}^{-1}} \boldsymbol{\Sigma} \mathbf{P}_{C_{2}^{\prime} ; S_{2}^{-1}}^{\prime}\right]
$$

is introduced since via $\mathbf{L}^{\prime}\left(\mathbf{C}_{2} \mathbf{C}_{2}^{\prime}\right)^{-} \mathbf{C}_{2} \mathbf{F}_{1} \mathbf{C}_{2}^{\prime}\left(\mathbf{C}_{2} \mathbf{C}_{2}^{\prime}\right)^{-} \mathbf{L}$ the expectation in (10) is obtained. Observe, that $\mathbf{S}_{2}$ can be rewritten as

$$
\mathbf{S}_{2}=\mathbf{S}_{1}+\mathbf{S}_{1} \mathbf{C}_{3}^{\prime o}\left(\mathbf{C}_{3}^{\prime o^{\prime}} \mathbf{S}_{1} \mathbf{C}_{3}^{\prime o}\right)^{-} \mathbf{C}_{3}^{\prime o^{\prime}} \mathbf{Y} \mathbf{P}_{Q_{\left(A_{1}: A_{2}\right)} A_{3}} \mathbf{Y}^{\prime} \mathbf{C}_{3}^{\prime o}\left(\mathbf{C}_{3}^{\prime o^{\prime}} \mathbf{S}_{1} \mathbf{C}_{3}^{\prime o}\right)^{-} \mathbf{C}_{3}^{\prime o^{\prime}} \mathbf{S}_{1}
$$

and, following Kollo and von Rosen (2005) [p. 376], formula (11) can be expressed as

$$
\begin{aligned}
& \mathbf{F}_{1}=E\left[\left(\mathbf{I}_{p}-\mathbf{S}_{2} \mathbf{C}_{2}^{\prime o}\left(\mathbf{C}_{2}^{\prime o} \mathbf{S}_{2} \mathbf{C}_{2}^{\prime o}\right)^{-} \mathbf{C}_{2}^{\prime o}\right) \boldsymbol{\Sigma}\left(\mathbf{I}_{p}-\mathbf{C}_{2}^{\prime o}\left(\mathbf{C}_{2}^{\prime o} \mathbf{S}_{2} \mathbf{C}_{2}^{\prime o}\right)^{-} \mathbf{C}_{2}^{\prime o} \mathbf{S}_{2}\right)\right] \\
& =\boldsymbol{\Sigma}-E\left[\mathbf{S}_{2} \mathbf{C}_{2}^{\prime o}\left(\mathbf{C}_{2}^{\prime o^{\prime}} \mathbf{S}_{2} \mathbf{C}_{2}^{\prime o}\right)^{-} \mathbf{C}_{2}^{\prime o^{\prime}} \mathbf{\Sigma}\right]-E\left[\boldsymbol{\Sigma} \mathbf{C}_{2}^{\prime o}\left(\mathbf{C}_{2}^{\prime o} \mathbf{S}_{2} \mathbf{C}_{2}^{\prime o}\right)^{-} \mathbf{C}_{2}^{\prime o} \mathbf{S}_{2}\right] \\
& +E\left[\mathbf{S}_{2} \mathbf{C}_{2}^{\prime o}\left(\mathbf{C}_{2}^{\prime o^{\prime}} \mathbf{S}_{2} \mathbf{C}_{2}^{\prime o}\right)^{-} \mathbf{C}_{2}^{\prime o^{\prime}} \boldsymbol{\Sigma} \mathbf{C}_{2}^{\prime o}\left(\mathbf{C}_{2}^{\prime o} \mathbf{S}_{2} \mathbf{C}_{2}^{\prime o}\right)^{-} \mathbf{C}_{2}^{\prime o} \mathbf{S}_{2}\right] \\
& =\boldsymbol{\Sigma}-\boldsymbol{\Sigma}^{1 / 2} E\left[\boldsymbol{\Sigma}^{-1 / 2} \mathbf{S}_{2} \mathbf{C}_{2}^{\prime o}\left(\mathbf{C}_{2}^{\prime o} \mathbf{S}_{2} \mathbf{C}_{2}^{\prime o}\right)^{-} \mathbf{C}_{2}^{\prime o^{\prime}} \boldsymbol{\Sigma}^{1 / 2}\right] \boldsymbol{\Sigma}^{1 / 2} \\
& -\boldsymbol{\Sigma}^{1 / 2} E\left[\boldsymbol{\Sigma}^{1 / 2} \mathbf{C}_{2}^{\prime o}\left(\mathbf{C}_{2}^{\prime o} \mathbf{S}_{2} \mathbf{C}_{2}^{\prime o}\right)^{-} \mathbf{C}_{2}^{\prime o^{\prime}} \mathbf{S}_{2} \boldsymbol{\Sigma}^{-1 / 2}\right] \boldsymbol{\Sigma}^{1 / 2} \\
& +\boldsymbol{\Sigma}^{1 / 2} E\left[\boldsymbol{\Sigma}^{-1 / 2} \mathbf{S}_{2} \mathbf{C}_{2}^{\prime o}\left(\mathbf{C}_{2}^{\prime o} \mathbf{S}_{2} \mathbf{C}_{2}^{o}\right)^{-} \mathbf{C}_{2}^{\prime o} \boldsymbol{\Sigma}^{1 / 2} \boldsymbol{\Sigma}^{1 / 2} \mathbf{C}_{2}^{\prime o}\left(\mathbf{C}_{2}^{\prime o} \mathbf{S}_{2} \mathbf{C}_{2}^{\prime o}\right)^{-}\right. \\
& \left.\times \mathbf{C}_{2}^{\prime o^{\prime}} \mathbf{S}_{2} \boldsymbol{\Sigma}^{-1 / 2}\right] \Sigma^{1 / 2} \text {. }
\end{aligned}
$$

Put

$$
\begin{aligned}
& \mathbf{V}_{1}=\Sigma^{-1 / 2} \mathbf{S}_{1} \Sigma^{-1 / 2}, \quad \mathbf{D}_{2}^{o}=\Sigma^{1 / 2} \mathbf{C}_{2}^{o}, \quad \mathbf{Z}=\mathbf{Y} \boldsymbol{\Sigma}^{-1 / 2}, \quad \mathbf{D}_{3}^{o}=\Sigma^{1 / 2} \mathbf{C}_{3}^{\prime o}, \\
& \mathbf{V}_{2}=\mathbf{V}_{1}+\mathbf{V}_{1} \mathbf{D}_{3}^{o}\left(\mathbf{D}_{3}^{o^{\prime}} \mathbf{V}_{1} \mathbf{D}_{3}^{o}\right)^{-} \mathbf{D}_{3}^{o^{\prime}} \mathbf{Z}^{\prime} \mathbf{P}_{Q_{\left(A_{1}: A_{2}\right)} A_{3}} \mathbf{Z} \mathbf{D}_{3}^{o}\left(\mathbf{D}_{3}^{o^{\prime}} \mathbf{V}_{1} \mathbf{D}_{3}^{o}\right)^{-} \mathbf{D}_{3}^{o^{\prime}} \mathbf{V}_{1} .
\end{aligned}
$$

Then, since $\mathcal{R}\left(\mathbf{D}_{2}\right)^{\perp} \subseteq \mathcal{R}\left(\mathbf{D}_{3}\right)^{\perp}$ Eq. (12) can be written as

$$
\begin{aligned}
\mathbf{F}_{1}= & \boldsymbol{\Sigma}-\boldsymbol{\Sigma}^{1 / 2} E\left[\mathbf{V}_{2} \mathbf{D}_{2}^{o}\left(\mathbf{D}_{2}^{o^{\prime}} \mathbf{V}_{2} \mathbf{D}_{2}^{o}\right)^{-} \mathbf{D}_{2}^{o^{\prime}}\right] \boldsymbol{\Sigma}^{1 / 2}-\boldsymbol{\Sigma}^{1 / 2} E\left[\mathbf{D}_{2}^{o}\left(\mathbf{D}_{2}^{o^{\prime}} \mathbf{V}_{2} \mathbf{D}_{2}^{o}\right)^{-} \mathbf{D}_{2}^{o^{\prime}} \mathbf{V}_{2}\right] \boldsymbol{\Sigma}^{1 / 2} \\
& +\boldsymbol{\Sigma}^{1 / 2} E\left[\mathbf{V}_{2} \mathbf{D}_{2}^{o}\left(\mathbf{D}_{2}^{o} \mathbf{V}_{2} \mathbf{D}_{2}^{o}\right)^{-} \mathbf{D}_{2}^{o^{\prime}} \mathbf{D}_{2}^{o}\left(\mathbf{D}_{2}^{o^{\prime}} \mathbf{V}_{2} \mathbf{D}_{2}^{o}\right)^{-} \mathbf{D}_{2}^{o^{\prime}} \mathbf{V}_{2}\right] \boldsymbol{\Sigma}^{1 / 2} \\
= & \boldsymbol{\Sigma}-\boldsymbol{\Sigma}^{1 / 2} E\left[\mathbf{T}_{1}\right] \Sigma^{1 / 2}-\boldsymbol{\Sigma}^{1 / 2} E\left[\mathbf{T}_{1}^{\prime}\right] \Sigma^{1 / 2}+\boldsymbol{\Sigma}^{1 / 2} E\left[\mathbf{T}_{1} \mathbf{T}_{1}^{\prime}\right] \Sigma^{1 / 2}
\end{aligned}
$$

where $\mathbf{T}_{1}=\mathbf{V}_{2} \mathbf{D}_{2}^{o}\left(\mathbf{D}_{2}^{o^{\prime}} \mathbf{V}_{2} \mathbf{D}_{2}^{o}\right)^{-} \mathbf{D}_{2}^{o^{\prime}}$.

Firstly $E\left[\mathbf{T}_{1}\right]$ is obtained. Since $\mathbf{I}_{p}=\mathbf{P}_{D_{3}^{o}}+\mathbf{P}_{D_{3}}$

$$
E\left[\mathbf{T}_{1}\right]=\mathbf{P}_{D_{3}^{o}} E\left[\mathbf{T}_{1}\right]+\mathbf{P}_{D_{3}} E\left[\mathbf{T}_{1}\right]
$$


Using $\mathcal{R}\left(\mathbf{D}_{2}\right)^{\perp} \subseteq \mathcal{R}\left(\mathbf{D}_{3}\right)^{\perp}$ it can be observed that

$$
\begin{aligned}
\mathbf{D}_{3}^{o^{\prime}} \mathbf{V}_{2} \mathbf{D}_{2}^{o}= & \mathbf{D}_{3}^{o^{\prime}} \mathbf{V}_{1} \mathbf{D}_{2}^{o}+\mathbf{D}_{3}^{o^{\prime}} \mathbf{V}_{1} \mathbf{D}_{3}^{o}\left(\mathbf{D}_{3}^{o^{\prime}} \mathbf{V}_{1} \mathbf{D}_{3}^{o}\right)^{-} \mathbf{D}_{3}^{o^{\prime}} \mathbf{Z}^{\prime} \mathbf{P}_{Q_{\left(A_{1}: A_{2}\right)} A_{3}} \mathbf{Z} \mathbf{D}_{3}^{o} \\
& \times\left(\mathbf{D}_{3}^{o^{\prime}} \mathbf{V}_{1} \mathbf{D}_{3}^{o}\right)^{-} \mathbf{D}_{3}^{o^{\prime}} \mathbf{V}_{1} \mathbf{D}_{2}^{o} \\
= & \mathbf{D}_{3}^{o^{\prime}}\left(\mathbf{V}_{1}+\mathbf{Z}^{\prime} \mathbf{P}_{Q_{\left(A_{1}: A_{2}\right)} A_{3}} \mathbf{Z}\right) \mathbf{D}_{2}^{o} \equiv \mathbf{D}_{3}^{o^{\prime}} \mathbf{W}_{2} \mathbf{D}_{2}^{o}
\end{aligned}
$$

and similarly

$$
\mathbf{D}_{2}^{o^{\prime}} \mathbf{V}_{2} \mathbf{D}_{2}^{o}=\mathbf{D}_{2}^{o^{\prime}} \mathbf{W}_{2} \mathbf{D}_{2}^{o},
$$

where because of independence between $\mathbf{V}_{1}$ and $\mathbf{Z}^{\prime} \mathbf{P}_{Q_{\left(A_{1}: A_{2}\right)} A_{3}} \mathbf{Z}$

$$
\mathbf{W}_{2} \sim \mathcal{W}_{p}\left(\mathbf{I}_{p}, n-\operatorname{rank}\left(\mathbf{A}_{1}: \mathbf{A}_{2}\right)\right)
$$

Let us in (14) determine $\mathbf{P}_{D_{3}^{o}} E\left[\mathbf{T}_{1}\right]$ :

$$
\begin{aligned}
& \mathbf{P}_{D_{3}^{o}} E\left[\mathbf{T}_{1}\right]=\mathbf{D}_{3}^{o}\left(\mathbf{D}_{3}^{o^{\prime}} \mathbf{D}_{3}^{o}\right)^{-} E\left[\mathbf{D}_{3}^{o^{\prime}} \mathbf{V}_{2} \mathbf{D}_{2}^{o}\left(\mathbf{D}_{2}^{o^{\prime}} \mathbf{V}_{2} \mathbf{D}_{2}^{o}\right)^{-} \mathbf{D}_{2}^{o^{\prime}}\right] \\
& =\mathbf{D}_{3}^{o}\left(\mathbf{D}_{3}^{o^{\prime}} \mathbf{D}_{3}^{o}\right)^{-} E\left[\mathbf{D}_{3}^{o^{\prime}} \mathbf{W}_{2} \mathbf{D}_{2}^{o}\left(\mathbf{D}_{2}^{o^{\prime}} \mathbf{W}_{2} \mathbf{D}_{2}^{o}\right)^{-} \mathbf{D}_{2}^{o^{\prime}}\right] \\
& =\mathbf{P}_{D_{3}^{o}} E\left[\mathbf{W}_{2} \mathbf{D}_{2}^{o}\left(\mathbf{D}_{2}^{o^{\prime}} \mathbf{W}_{2} \mathbf{D}_{2}^{o}\right)^{-} \mathbf{D}_{2}^{o^{\prime}}\right] \\
& =\mathbf{P}_{D_{3}^{o}} \mathbf{P}_{D_{2}^{o}}=\mathbf{P}_{D_{2}^{o}}=\mathbf{P}_{\Sigma^{1 / 2} C_{2}^{\prime o}}
\end{aligned}
$$

(for the last taken expectation see Kollo and von Rosen 2005 [Problem 1, p. 275] with $D\left[\mathbf{W}_{2}\right]=\mathbf{I}_{p}$ ). Since $\mathbf{P}_{\Sigma^{1 / 2} X^{o}}=\mathbf{I}_{p}-\mathbf{P}_{\Sigma^{-1 / 2} X}$ (see e.g. Markiewicz 2001), we may write

$$
\mathbf{P}_{D_{3}^{o}} E\left[\mathbf{T}_{1}\right]=\mathbf{I}_{p}-\mathbf{P}_{\Sigma^{-1 / 2} C_{2}^{\prime}}
$$

Moreover, in (14) consider $\mathbf{P}_{D_{3}} E\left[\mathbf{T}_{1}\right]$. Since $\mathbf{V}_{1}$ is Wishart distributed we can factorize it as $\mathbf{V}_{1}=\mathbf{X} \mathbf{X}^{\prime}$, where $\mathbf{X} \sim N_{p, n}\left(\mathbf{0}, \mathbf{I}_{p}, \mathbf{I}_{n}\right)$. Furthermore, since $\mathbf{D}_{3}^{\prime}\left(\mathbf{D}_{2}^{o}: \mathbf{D}_{3}^{o}\right)=\mathbf{0}$ we have that $\mathbf{D}_{3}^{\prime} \mathbf{X}$ is independent of $\mathbf{D}_{2}^{o^{\prime}} \mathbf{X}$ and $\mathbf{D}_{3}^{o^{\prime}} \mathbf{X}$, and hence

$$
\begin{aligned}
& \mathbf{P}_{D_{3}} E\left[\mathbf{T}_{1}\right]=\mathbf{D}_{3}\left(\mathbf{D}_{3}^{\prime} \mathbf{D}_{3}\right)^{-} E\left[\mathbf{D}_{3}^{\prime} \mathbf{V}_{2} \mathbf{D}_{2}^{o}\left(\mathbf{D}_{2}^{o^{\prime}} \mathbf{V}_{2} \mathbf{D}_{2}^{o}\right)^{-} \mathbf{D}_{2}^{o^{\prime}}\right] \\
& =\mathbf{D}_{3}\left(\mathbf{D}_{3}^{\prime} \mathbf{D}_{3}\right)^{-} E\left[\mathbf{D}_{3}^{\prime} \mathbf{V}_{1} \mathbf{D}_{2}^{o}\left(\mathbf{D}_{2}^{o^{\prime}} \mathbf{W}_{2} \mathbf{D}_{2}^{o}\right)^{-} \mathbf{D}_{2}^{o^{\prime}}\right. \\
& +\mathbf{D}_{3}^{\prime} \mathbf{V}_{1} \mathbf{D}_{3}^{o}\left(\mathbf{D}_{3}^{o^{\prime}} \mathbf{V}_{1} \mathbf{D}_{3}^{o}\right)^{-} \mathbf{D}_{3}^{o^{\prime}} \mathbf{Z}^{\prime} \mathbf{P}_{Q_{\left(A_{1}: A_{2}\right)} A_{3}} \mathbf{Z D}_{3}^{o}\left(\mathbf{D}_{3}^{o^{\prime}} \mathbf{V}_{1} \mathbf{D}_{3}^{o}\right)^{-} \\
& \left.\times \mathbf{D}_{3}^{o^{\prime}} \mathbf{V}_{1} \mathbf{D}_{2}^{o}\left(\mathbf{D}_{2}^{o^{\prime}} \mathbf{W}_{2} \mathbf{D}_{2}^{o}\right)^{-} \mathbf{D}_{2}^{o^{\prime}}\right] \\
& =\mathbf{D}_{3}\left(\mathbf{D}_{3}^{\prime} \mathbf{D}_{3}\right)^{-} E\left[\mathbf { D } _ { 3 } ^ { \prime } \mathbf { X } \left(\mathbf{I}_{n}+\mathbf{X}^{\prime} \mathbf{D}_{3}^{o}\left(\mathbf{D}_{3}^{o^{\prime}} \mathbf{V}_{1} \mathbf{D}_{3}^{o}\right)^{-} \mathbf{D}_{3}^{o^{\prime}} \mathbf{Z}^{\prime} \mathbf{P}_{Q_{\left(A_{1}: A_{2}\right)} A_{3}} \mathbf{Z}\right.\right. \\
& \left.\left.\times \mathbf{D}_{3}^{o}\left(\mathbf{D}_{3}^{o^{\prime}} \mathbf{V}_{1} \mathbf{D}_{3}^{o}\right)^{-} \mathbf{D}_{3}^{o^{\prime}} \mathbf{X}\right) \mathbf{X}^{\prime} \mathbf{D}_{2}^{o}\left(\mathbf{D}_{2}^{o^{\prime}} \mathbf{W}_{2} \mathbf{D}_{2}^{o}\right)^{-} \mathbf{D}_{2}^{o^{\prime}}\right] \\
& =\mathbf{D}_{3}\left(\mathbf{D}_{3}^{\prime} \mathbf{D}_{3}\right)^{-} \underbrace{E\left[\mathbf{D}_{3}^{\prime} \mathbf{X}\right]}_{\mathbf{0}}
\end{aligned}
$$




$$
\begin{aligned}
& \times E\left[\left(\mathbf{I}_{n}+\mathbf{X}^{\prime} \mathbf{D}_{3}^{o}\left(\mathbf{D}_{3}^{o^{\prime}} \mathbf{V}_{1} \mathbf{D}_{3}^{o}\right)^{-} \mathbf{D}_{3}^{o^{\prime}} \mathbf{Z}^{\prime} \mathbf{P}_{Q_{\left(A_{1}: A_{2}\right)} A_{3}} \mathbf{Z} \mathbf{D}_{3}^{o}\right.\right. \\
& \left.\left.\times\left(\mathbf{D}_{3}^{o^{\prime}} \mathbf{V}_{1} \mathbf{D}_{3}^{o}\right)^{-} \mathbf{D}_{3}^{o^{\prime}} \mathbf{X}\right) \mathbf{X}^{\prime} \mathbf{D}_{2}^{o}\left(\mathbf{D}_{2}^{o^{\prime}} \mathbf{W}_{2} \mathbf{D}_{2}^{o}\right)^{-} \mathbf{D}_{2}^{o^{\prime}}\right]=\mathbf{0}
\end{aligned}
$$

Thus, $E\left[\mathbf{T}_{1}\right]=\mathbf{I}_{p}-\mathbf{P}_{\Sigma^{-1 / 2} C_{2}^{\prime}}$.

In the next we consider $E\left[\mathbf{T}_{1} \mathbf{T}_{1}^{\prime}\right]$. Since again $\mathbf{I}_{p}=\mathbf{P}_{D_{3}^{o}}+\mathbf{P}_{D_{3}}$ and since $\mathbf{D}_{3}^{\prime} \mathbf{X}$ is independent of $\mathbf{D}_{2}^{o^{\prime}} \mathbf{X}$ and $\mathbf{D}_{3}^{o^{\prime}} \mathbf{X}$, we will calculate

$$
\left(\mathbf{P}_{D_{3}^{o}}+\mathbf{P}_{D_{3}}\right) E\left[\mathbf{T}_{1} \mathbf{T}_{1}^{\prime}\right]\left(\mathbf{P}_{D_{3}^{o}}+\mathbf{P}_{D_{3}}\right)=\mathbf{P}_{D_{3}^{o}} E\left[\mathbf{T}_{1} \mathbf{T}_{1}^{\prime}\right] \mathbf{P}_{D_{3}^{o}}+\mathbf{P}_{D_{3}} E\left[\mathbf{T}_{1} \mathbf{T}_{1}^{\prime}\right] \mathbf{P}_{D_{3}},
$$

because $\mathbf{P}_{D_{3}^{o}} E\left[\mathbf{T}_{1} \mathbf{T}_{1}^{\prime}\right] \mathbf{P}_{D_{3}}=\mathbf{0}$. Using (15) and (16) we can write

$$
\begin{aligned}
\mathbf{P}_{D_{3}^{o}} E\left[\mathbf{T}_{1} \mathbf{T}_{1}^{\prime}\right] \mathbf{P}_{D_{3}^{o}}= & \mathbf{D}_{3}^{o}\left(\mathbf{D}_{3}^{o^{\prime}} \mathbf{D}_{3}^{o}\right)^{-} E\left[\mathbf{D}_{3}^{o^{\prime}} \mathbf{V}_{2} \mathbf{D}_{2}^{o}\left(\mathbf{D}_{2}^{o^{\prime}} \mathbf{V}_{2} \mathbf{D}_{2}^{o}\right)^{-} \mathbf{D}_{2}^{o^{\prime}} \mathbf{D}_{2}^{o}\left(\mathbf{D}_{2}^{o^{\prime}} \mathbf{V}_{2} \mathbf{D}_{2}^{o}\right)^{-} \mathbf{D}_{2}^{o^{\prime}} \mathbf{V}_{2} \mathbf{D}_{3}^{o}\right] \\
& \times\left(\mathbf{D}_{3}^{o^{\prime}} \mathbf{D}_{3}^{o}\right)^{-} \mathbf{D}_{3}^{o^{\prime}} \\
= & \mathbf{P}_{D_{3}^{o}} E\left[\mathbf{W}_{2} \mathbf{D}_{2}^{o}\left(\mathbf{D}_{2}^{o^{\prime}} \mathbf{W}_{2} \mathbf{D}_{2}^{o}\right)^{-} \mathbf{D}_{2}^{o^{\prime}} \mathbf{D}_{2}^{o}\left(\mathbf{D}_{2}^{o} \mathbf{W}_{2} \mathbf{D}_{2}^{o}\right)^{-} \mathbf{D}_{2}^{o^{\prime}} \mathbf{W}_{2}\right] \mathbf{P}_{D_{3}^{o}} .
\end{aligned}
$$

Observe, that the expression in the expectation may be expressed as formula (4.2.45) of Kollo and von Rosen (2005) [p. 419] and therefore, following Kollo and von Rosen (2005) [(4.2.48), (4.2.50), (4.2.51), (4.2.56), and (4.2.58), p. 419-420], we obtain

$$
\mathbf{P}_{D_{3}^{o}} E\left[\mathbf{T}_{1} \mathbf{T}_{1}^{\prime}\right] \mathbf{P}_{D_{3}^{o}}=\left(1-\gamma_{2}\right) \mathbf{P}_{\Sigma^{1 / 2} C_{2}^{\prime o}}+\gamma_{2} \mathbf{P}_{\Sigma^{1 / 2} C_{3}^{\prime o}}
$$

with $\gamma_{2}$ defined in the statement of the theorem. Moreover, since $\mathbf{P}_{\Sigma^{-1 / 2} C^{o}}=\mathbf{Q}_{\Sigma^{1 / 2} C}$ (cf. Markiewicz 2001; Kollo and von Rosen 2005),

$$
\mathbf{P}_{D_{3}^{o}} E\left[\mathbf{T}_{1} \mathbf{T}_{1}^{\prime}\right] \mathbf{P}_{D_{3}^{o}}=\mathbf{I}_{p}-\left(1-\gamma_{2}\right) \mathbf{P}_{\Sigma^{-1 / 2} C_{2}^{\prime}}-\gamma_{2} \mathbf{P}_{\Sigma^{-1 / 2} C_{3}^{\prime}} .
$$

In order to verify (ii) it remains to calculate $\mathbf{P}_{D_{3}} E\left[\mathbf{T}_{1} \mathbf{T}_{1}^{\prime}\right] \mathbf{P}_{D_{3}}$. Using $\mathbf{V}_{1}=\mathbf{X X} \mathbf{X}^{\prime}$

$$
\begin{aligned}
\mathbf{P}_{D_{3}} E\left[\mathbf{T}_{1} \mathbf{T}_{1}^{\prime}\right] \mathbf{P}_{D_{3}}= & \mathbf{D}_{3}\left(\mathbf{D}_{3}^{\prime} \mathbf{D}_{3}\right)^{-} E\left[\mathbf{D}_{3}^{\prime} \mathbf{V}_{2} \mathbf{D}_{2}^{o}\left(\mathbf{D}_{2}^{o^{\prime}} \mathbf{V}_{2} \mathbf{D}_{2}^{o}\right)^{-} \mathbf{D}_{2}^{o^{\prime}} \mathbf{D}_{2}^{o}\left(\mathbf{D}_{2}^{o^{\prime}} \mathbf{V}_{2} \mathbf{D}_{2}^{o}\right)^{-} \mathbf{D}_{2}^{o^{\prime}} \mathbf{V}_{2} \mathbf{D}_{3}\right] \\
& \times\left(\mathbf{D}_{3}^{\prime} \mathbf{D}_{3}\right)^{-} \mathbf{D}_{3}^{\prime} \\
= & \mathbf{D}_{3}\left(\mathbf{D}_{3}^{\prime} \mathbf{D}_{3}\right)^{-} E\left[\mathbf{D}_{3}^{\prime} \mathbf{X}\left(\mathbf{I}_{n}+\mathbf{X}^{\prime} \mathbf{\Lambda} \mathbf{X}\right) \mathbf{X}^{\prime} \mathbf{D}_{2}^{o}\left(\mathbf{D}_{2}^{o^{\prime}} \mathbf{W}_{2} \mathbf{D}_{2}^{o}\right)^{-} \mathbf{D}_{2}^{o^{\prime}}\right. \\
& \left.\left.\times \mathbf{D}_{2}^{o}\left(\mathbf{D}_{2}^{o^{\prime}} \mathbf{W}_{2} \mathbf{D}_{2}^{o}\right)^{-} \mathbf{D}_{2}^{o^{\prime}} \mathbf{X}\left(\mathbf{I}_{n}+\mathbf{X}^{\prime} \mathbf{\Lambda} \mathbf{X}\right)\right) \mathbf{X}^{\prime} \mathbf{D}_{3}\right]\left(\mathbf{D}_{3}^{\prime} \mathbf{D}_{3}\right)^{-} \mathbf{D}_{3}^{\prime}
\end{aligned}
$$


with $\boldsymbol{\Lambda}=\mathbf{D}_{3}^{o}\left(\mathbf{D}_{3}^{o^{\prime}} \mathbf{V}_{1} \mathbf{D}_{3}^{o}\right)^{-} \mathbf{D}_{3}^{o^{\prime}} \mathbf{Z}^{\prime} \mathbf{P}_{Q_{\left(A_{1}: A_{2}\right)} A_{3}} \mathbf{Z} \mathbf{D}_{3}^{o}\left(\mathbf{D}_{3}^{o^{\prime}} \mathbf{V}_{1} \mathbf{D}_{3}^{o}\right)^{-} \mathbf{D}_{3}^{o^{\prime}}$ and using the independence of $\mathbf{D}_{3}^{\prime} \mathbf{X}$ with $\mathbf{D}_{2}^{o^{\prime}} \mathbf{X}$ and $\mathbf{D}_{3}^{o^{\prime}} \mathbf{X}$, we may calculate conditional expectation. Hence, using Theorem 2.2.9 (i) of Kollo and von Rosen (2005) [p. 207], we may write

$$
\begin{array}{rl}
\mathbf{P}_{D_{3}} E & E\left[\mathbf{T}_{1} \mathbf{T}_{1}^{\prime}\right] \mathbf{P}_{D_{3}} \\
= & E\left[\operatorname{tr}\left\{\left(\mathbf{I}_{n}+\mathbf{X}^{\prime} \mathbf{\Lambda} \mathbf{X}\right) \mathbf{X}^{\prime} \mathbf{D}_{2}^{o}\left(\mathbf{D}_{2}^{o^{\prime}} \mathbf{W}_{2} \mathbf{D}_{2}^{o}\right)^{-} \mathbf{D}_{2}^{o^{\prime}} \mathbf{D}_{2}^{o}\left(\mathbf{D}_{2}^{o^{\prime}} \mathbf{W}_{2} \mathbf{D}_{2}^{o}\right)^{-} \mathbf{D}_{2}^{o^{\prime}} \mathbf{X}\left(\mathbf{I}_{n}+\mathbf{X}^{\prime} \boldsymbol{\Lambda} \mathbf{X}\right)\right\}\right] \mathbf{P}_{D_{3}} \\
= & E\left[\operatorname { t r } \left\{\left(\mathbf{X}^{\prime} \mathbf{X}\right)^{-1} \mathbf{X}^{\prime} \mathbf{X}\left(\mathbf{X}^{\prime}+\mathbf{X}^{\prime} \mathbf{\Lambda} \mathbf{X} \mathbf{X}^{\prime}\right) \mathbf{D}_{2}^{o}\left(\mathbf{D}_{2}^{o^{\prime}} \mathbf{W}_{2} \mathbf{D}_{2}^{o}\right)^{-} \mathbf{D}_{2}^{o^{\prime}} \mathbf{D}_{2}^{o}\left(\mathbf{D}_{2}^{o^{\prime}} \mathbf{W}_{2} \mathbf{D}_{2}^{o}\right)^{-} \mathbf{D}_{2}^{o^{\prime}}\right.\right. \\
& \left.\left.\times\left(\mathbf{X}+\mathbf{X} \mathbf{X}^{\prime} \mathbf{\Lambda} \mathbf{X}\right) \mathbf{X}^{\prime} \mathbf{X}\left(\mathbf{X}^{\prime} \mathbf{X}\right)^{-1}\right\}\right] \mathbf{P}_{D_{3}} \\
= & E\left[\operatorname{tr}\left\{\mathbf{V}_{2} \mathbf{D}_{2}^{o}\left(\mathbf{D}_{2}^{o^{\prime}} \mathbf{W}_{2} \mathbf{D}_{2}^{o}\right)^{-} \mathbf{D}_{2}^{o^{\prime}} \mathbf{D}_{2}^{o}\left(\mathbf{D}_{2}^{o} \mathbf{W}_{2} \mathbf{D}_{2}^{o}\right)^{-} \mathbf{D}_{2}^{o^{\prime}} \mathbf{V}_{2} \mathbf{V}_{1}^{-1}\right\}\right] \mathbf{P}_{D_{3} .} .
\end{array}
$$

Moreover, $\mathbf{V}_{1}^{-1}=\mathbf{V}_{1}^{-1} \mathbf{D}_{3}\left(\mathbf{D}_{3}^{\prime} \mathbf{V}_{1}^{-1} \mathbf{D}_{3}\right)^{-} \mathbf{D}_{3}^{\prime} \mathbf{V}_{1}^{-1}+\mathbf{D}_{3}^{o}\left(\mathbf{D}_{3}^{o^{\prime}} \mathbf{V}_{1} \mathbf{D}_{3}^{o}\right)^{-} \mathbf{D}_{3}^{o^{\prime}}$. Since $\mathbf{D}_{3}^{\prime} \mathbf{V}_{1}^{-1} \mathbf{V}_{2} \mathbf{D}_{2}^{o}=\mathbf{0}$, we get

$$
\begin{aligned}
\mathbf{P}_{D_{3}} E\left[\mathbf{T}_{1} \mathbf{T}_{1}^{\prime}\right] \mathbf{P}_{D_{3}}=E & {\left[\operatorname { t r } \left\{\mathbf{D}_{3}^{o^{\prime}} \mathbf{W}_{2} \mathbf{D}_{2}^{o}\left(\mathbf{D}_{2}^{o^{\prime}} \mathbf{W}_{2} \mathbf{D}_{2}^{o}\right)^{-} \mathbf{D}_{2}^{o^{\prime}} \mathbf{D}_{2}^{o}\right.\right.} \\
& \left.\left.\times\left(\mathbf{D}_{2}^{o^{\prime}} \mathbf{W}_{2} \mathbf{D}_{2}^{o}\right)^{-} \mathbf{D}_{2}^{o^{\prime}} \mathbf{W}_{2} \mathbf{D}_{3}^{o}\left(\mathbf{D}_{3}^{o^{\prime}} \mathbf{V}_{1} \mathbf{D}_{3}^{o}\right)^{-}\right\}\right] \mathbf{P}_{D_{3}} .
\end{aligned}
$$

Without loss of generality from now on we identify $\mathbf{D}_{2}^{o}$ and $\mathbf{D}_{3}^{o}$ with matrices of full rank, i.e., $\mathbf{D}_{2}^{o}: p \times\left(p-\operatorname{rank}\left(\mathbf{D}_{2}\right)\right)$ and $\mathbf{D}_{3}^{o}: p \times\left(p-\operatorname{rank}\left(\mathbf{D}_{3}\right)\right)$. We are going to rewrite (18) in a canonical form and use that there exist a non-singular matrix $\mathbf{M}:\left(p-\operatorname{rank}\left(\mathbf{D}_{3}\right)\right) \times\left(p-\operatorname{rank}\left(\mathbf{D}_{3}\right)\right)$ and an orthogonal matrix $\boldsymbol{\Gamma}: p \times p$ such that

$$
\mathbf{D}_{3}^{o^{\prime}}=\mathbf{M}\left(\mathbf{I}_{r}: \mathbf{0}\right) \boldsymbol{\Gamma}, \quad r=p-\operatorname{rank}\left(\mathbf{D}_{3}\right)=p-\operatorname{rank}\left(\mathbf{C}_{3}\right)
$$

and since $\mathcal{R}\left(\mathbf{D}_{2}\right)^{\perp} \subseteq \mathcal{R}\left(\mathbf{D}_{3}\right)^{\perp}$ there exists a matrix $\mathbf{Q}$ such that

$$
\mathbf{D}_{2}^{o}=\boldsymbol{\Gamma}^{\prime}\left(\mathbf{I}_{r}: \mathbf{0}\right)^{\prime} \mathbf{M}^{\prime} \mathbf{Q}
$$

Therefore the trace in (18) equals

$$
\begin{aligned}
\operatorname{tr}\left\{\mathbf{W}_{11}\right. & \mathbf{M}^{\prime} \mathbf{Q}\left(\mathbf{Q}^{\prime} \mathbf{M} \mathbf{W}_{11} \mathbf{M}^{\prime} \mathbf{Q}\right)^{-1} \mathbf{Q}^{\prime} \mathbf{M} \\
& \left.\times \mathbf{M}^{\prime} \mathbf{Q}\left(\mathbf{Q}^{\prime} \mathbf{M} \mathbf{W}_{11} \mathbf{M}^{\prime} \mathbf{Q}\right)^{-1} \mathbf{Q}^{\prime} \mathbf{M} \mathbf{W}_{11}\left(\mathbf{V}_{11}\right)^{-1}\right\}
\end{aligned}
$$

where $\mathbf{W}_{11}=\left(\mathbf{I}_{r}: \mathbf{0}\right) \boldsymbol{\Gamma} \mathbf{W}_{2} \boldsymbol{\Gamma}^{\prime}\left(\mathbf{I}_{r}: \mathbf{0}\right)^{\prime}$ and $\mathbf{V}_{11}=\left(\mathbf{I}_{r}: \mathbf{0}\right) \boldsymbol{\Gamma} \mathbf{V}_{1} \boldsymbol{\Gamma}^{\prime}\left(\mathbf{I}_{r}: \mathbf{0}\right)^{\prime}$. We may also express the above trace as

$$
\begin{aligned}
\operatorname{tr}\left\{\mathbf{M}^{\prime} \mathbf{Q}\right. & \left(\mathbf{Q}^{\prime} \mathbf{M} \mathbf{W}_{11} \mathbf{M}^{\prime} \mathbf{Q}\right)^{-1} \mathbf{Q}^{\prime} \mathbf{M} \mathbf{W}_{11}^{1 / 2} \mathbf{W}_{11}^{1 / 2} \mathbf{V}_{11}^{-1} \mathbf{W}_{11}^{1 / 2} \\
\times & \left.\mathbf{W}_{11}^{1 / 2} \mathbf{M}^{\prime} \mathbf{Q}\left(\mathbf{Q}^{\prime} \mathbf{M} \mathbf{W}_{11} \mathbf{M}^{\prime} \mathbf{Q}\right)^{-1} \mathbf{Q}^{\prime} \mathbf{M}\right\}
\end{aligned}
$$


Put in (19)

$$
\mathbf{N}=\mathbf{W}_{11}^{1 / 2}\left(\mathbf{V}_{11}\right)^{-1} \mathbf{W}_{11}^{1 / 2}
$$

which by Lemma 1 (see Appendix A) follows an inverse multivariate beta type I distribution with the important fact that the distribution is independent of $\mathbf{W}_{11}$. Then

$$
E[\mathbf{N}]=\frac{n-\operatorname{rank}\left(A_{1}: A_{2}\right)-r-1}{n-\operatorname{rank}\left(A_{1}: A_{2}: A_{3}\right)-r-1} \mathbf{I}_{r},
$$

since $\mathbf{W}_{11} \sim W_{r}\left(\mathbf{I}_{r}, n-\operatorname{rank}\left(\mathbf{A}_{1}: \mathbf{A}_{2}\right)\right)$ and $\mathbf{V}_{11} \sim W_{r}\left(\mathbf{I}_{r}, n-\operatorname{rank}\left(\mathbf{A}_{1}: \mathbf{A}_{2}: \mathbf{A}_{3}\right)\right)$.

Hence,

$$
\begin{aligned}
\mathbf{P}_{D_{3}} E\left[\mathbf{T}_{1} \mathbf{T}_{1}^{\prime}\right] \mathbf{P}_{D_{3}} & =\frac{n-\operatorname{rank}\left(A_{1}: A_{2}\right)-r-1}{n-\operatorname{rank}\left(A_{1}: A_{2}: A_{3}\right)-r-1} E\left[\mathbf{M}^{\prime} \mathbf{Q}\left(\mathbf{Q}^{\prime} \mathbf{M} \mathbf{W}_{11} \mathbf{M}^{\prime} \mathbf{Q}\right)^{-1} \mathbf{Q}^{\prime} \mathbf{M}\right] P_{D_{3}} \\
& =\frac{n-\operatorname{rank}\left(A_{1}: A_{2}\right)-r-1}{n-\operatorname{rank}\left(A_{1}: A_{2}: A_{3}\right)-r-1} E\left[\mathbf{D}_{2}^{o}\left(\mathbf{D}_{2}^{o} \mathbf{W}_{2} \mathbf{D}_{2}^{o}\right)^{-} \mathbf{D}_{2}^{o^{\prime}}\right] P_{D_{3}} .
\end{aligned}
$$

Following Theorem 2.4.14 (iii) of Kollo and von Rosen (2005) [p. 257] we may write $E\left[\left(\mathbf{D}_{2}^{o^{\prime}} \mathbf{W}_{2} \mathbf{D}_{2}^{o}\right)^{-}\right]=\frac{1}{n-\operatorname{rank}\left(A_{1}: A_{2}\right)-p+\operatorname{rank}\left(C_{2}\right)-1}\left(\mathbf{D}_{2}^{o^{\prime}} \mathbf{D}_{2}^{o}\right)^{-}$and, using Theorem 2.4.13 (i) of Kollo and von Rosen (2005) [p. 255] and the fact that $\operatorname{tr}\left(\mathbf{A A}^{-}\right)=\operatorname{rank}(\mathbf{A})$ we obtain

$$
\mathbf{P}_{D_{3}} E\left[\mathbf{T}_{1} \mathbf{T}_{1}^{\prime}\right] \mathbf{P}_{D_{3}}=\gamma_{3} \mathbf{P}_{\Sigma^{-1 / 2} C_{3}^{\prime}}
$$

since $\mathbf{D}_{3}=\boldsymbol{\Sigma}^{-1 / 2} \mathbf{C}_{3}^{\prime}$ and $\gamma_{3}$ is defined in the formulation of the theorem. Thus, due to (17) and (20)

$$
\begin{aligned}
E\left[\mathbf{T}_{1} \mathbf{T}_{1}^{\prime}\right] & =\left(1-\gamma_{2}\right)\left(\mathbf{I}_{p}-\mathbf{P}_{\Sigma^{-1 / 2} C_{2}^{\prime}}\right)+\gamma_{2}\left(\mathbf{I}_{p}-\mathbf{P}_{\Sigma^{-1 / 2} C_{3}^{\prime}}\right)+\gamma_{3} \mathbf{P}_{\Sigma^{-1 / 2} C_{3}^{\prime}} \\
& =\mathbf{I}_{p}-\left(1-\gamma_{2}\right) \mathbf{P}_{\Sigma^{-1 / 2} C_{2}^{\prime}}+\left(\gamma_{3}-\gamma_{2}\right) \mathbf{P}_{\Sigma^{-1 / 2} C_{3}^{\prime}}
\end{aligned}
$$

and

$$
\mathbf{F}_{1}=\boldsymbol{\Sigma}^{1 / 2}\left[\left(1+\gamma_{2}\right) \mathbf{P}_{\Sigma^{-1 / 2} C_{2}^{\prime}}+\left(\gamma_{3}-\gamma_{2}\right) \mathbf{P}_{\Sigma^{-1 / 2} C_{3}^{\prime}}\right] \boldsymbol{\Sigma}^{1 / 2}
$$

From (9) and (22) statement (ii) of the theorem is obtained.

Next theorems present $E(n \widehat{\mathbf{\Sigma}})$ for Model I and II, respectively.

Theorem 7 For Model I let $\widehat{\boldsymbol{\Sigma}}$ be given in Theorem 1 . Then,

$$
\begin{aligned}
E[n \widehat{\mathbf{\Sigma}}]=( & \left.n-\operatorname{rank}\left(\mathbf{A}_{1}: \mathbf{A}_{2}: \mathbf{A}_{3}\right)\right) \boldsymbol{\Sigma} \\
& +\left(\operatorname{rank}\left(\mathbf{A}_{1}: \mathbf{A}_{2}: \mathbf{A}_{3}\right)-\operatorname{rank}\left(\mathbf{A}_{1}: \mathbf{A}_{2}\right)\right)\left(\boldsymbol{\Sigma}-\left(1-\gamma_{9}\right) \mathbf{C}_{3}^{\prime}\left(\mathbf{C}_{3} \boldsymbol{\Sigma} \mathbf{C}_{3}^{\prime}\right)^{-} \mathbf{C}_{3}\right) \\
& +\left(\operatorname{rank}\left(\mathbf{A}_{1}: \mathbf{A}_{2}\right)-\operatorname{rank}\left(\mathbf{A}_{1}\right)\right) \boldsymbol{\Sigma}^{1 / 2} E\left[\mathbf{T}_{1} \mathbf{T}_{1}^{\prime}\right] \boldsymbol{\Sigma}^{1 / 2} \\
& +\operatorname{rank}\left(\mathbf{A}_{1}\right) \boldsymbol{\Sigma}^{1 / 2} E\left[\mathbf{T}_{2} \mathbf{T}_{2}^{\prime}\right] \boldsymbol{\Sigma}^{1 / 2}
\end{aligned}
$$


where $\gamma_{9}=\frac{p-\operatorname{rank}\left(C_{3}\right)}{\left.n-\operatorname{rank}\left(A_{1}: A_{2}: A_{3}\right)-p+\operatorname{rank}\left(C_{3}\right)\right)-1}$ and $E\left[\mathbf{T}_{1} \mathbf{T}_{1}^{\prime}\right]$ and $E\left[\mathbf{T}_{2} \mathbf{T}_{2}^{\prime}\right]$ are given by (21) and (B-19), respectively.

Proof The expression follows from the following calculations:

$$
\begin{aligned}
& E[n \widehat{\mathbf{\Sigma}}]= E\left[\mathbf{S}_{1}\right]+\operatorname{rank}\left(\mathbf{Q}_{\left(A_{1}: A_{2}\right)} \mathbf{A}_{3}\right) E\left[\mathbf{Q}_{C_{3}^{\prime} ; S_{1}^{-1}} \mathbf{\Sigma} \mathbf{Q}_{C_{3}^{\prime} ; S_{1}^{-1}}^{\prime}\right] \\
& \quad+\operatorname{rank}\left(\mathbf{Q}_{A_{1}} \mathbf{A}_{2}\right) E\left[\mathbf{Q}_{C_{2}^{\prime} ; S_{2}^{-1}} \mathbf{\Sigma} \mathbf{Q}_{C_{2}^{\prime} ; S_{2}^{-1}}^{\prime}\right]+\operatorname{rank}\left(\mathbf{A}_{1}\right) E\left[\mathbf{Q}_{C_{1} ; S_{3}^{-1}} \mathbf{\Sigma} \mathbf{Q}_{C_{1}^{\prime} ; S_{3}^{-1}}\right] \\
&=E\left[\mathbf{S}_{1}\right]+\operatorname{rank}\left(\mathbf{Q}_{\left(A_{1}: A_{2}\right)} \mathbf{A}_{3}\right) E\left[\mathbf{Q}_{C_{3}^{\prime} ; S_{1}^{-1}} \mathbf{\Sigma} \mathbf{Q}_{C_{3}^{\prime} ; S_{1}^{-1}}^{\prime}\right] \\
& \quad+\operatorname{rank}\left(\mathbf{Q}_{A_{1}} \mathbf{A}_{2}\right) \boldsymbol{\Sigma}^{1 / 2} E\left[\mathbf{T}_{1} \mathbf{T}_{1}^{\prime}\right] \boldsymbol{\Sigma}^{1 / 2}+\operatorname{rank}\left(\mathbf{A}_{1}\right) \boldsymbol{\Sigma}^{1 / 2} E\left[\mathbf{T}_{2} \mathbf{T}_{2}^{\prime}\right] \boldsymbol{\Sigma}^{1 / 2}
\end{aligned}
$$

and $E\left[\mathbf{Q}_{C_{3}^{\prime} ; S_{1}^{-1}} \boldsymbol{\Sigma} \mathbf{Q}_{C_{3}^{\prime} ; S_{1}^{-1}}^{\prime}\right]$ is obtained from Kollo and von Rosen (2005) [(4.2.45)(4.2.58), p. 419-420].

Theorem $\mathbf{8}$ For Model II let $\widehat{\mathbf{\Sigma}}$ be given in Theorem 2. Then,

$$
\begin{aligned}
E[n \widehat{\mathbf{\Sigma}}]=\boldsymbol{\Sigma}^{1 / 2}\left\{\left(n-\operatorname{rank}\left(\mathbf{A}_{1}\right)\right) \mathbf{I}_{p}+\left(\operatorname{rank}\left(\mathbf{A}_{1}\right)-\operatorname{rank}\left(\mathbf{A}_{2}\right)\right)\left(z_{11} \mathbf{K}_{1}+\mathbf{P}_{\Sigma^{1 / 2} G_{1}}\right)\right. \\
+\left(\operatorname{rank}\left(\mathbf{A}_{2}\right)-\operatorname{rank}\left(A_{3}\right)\right)\left(z_{12} \mathbf{K}_{1}+z_{22} \mathbf{K}_{2}+\mathbf{P}_{\Sigma^{1 / 2} G_{2}}\right) \\
\left.+\operatorname{rank}\left(\mathbf{A}_{3}\right)\left(z_{13} \mathbf{K}_{1}+z_{21} \mathbf{K}_{2}+z_{33} \mathbf{K}_{3}+\mathbf{P}_{\Sigma^{1 / 2} G_{3}}\right)\right\} \boldsymbol{\Sigma}^{1 / 2}
\end{aligned}
$$

where

$$
\begin{aligned}
v_{1} & =p-\operatorname{rank}\left(\mathbf{C}_{1}\right), \quad v_{i}=p-\operatorname{rank}\left(\mathbf{C}_{1}^{\prime}: \cdots: \mathbf{C}_{i}^{\prime}\right)+\operatorname{rank}\left(\mathbf{C}_{1}^{\prime}: \cdots: \mathbf{C}_{i-1}^{\prime}\right), \quad i=2,3, \\
k_{j} & =\frac{n-\operatorname{rank}\left(A_{j+1}\right)-v_{j}-1}{n-\operatorname{rank}\left(A_{j}\right)-v_{j}-1}, \quad j=1,2, \\
\mathbf{G}_{1} & =\left(\mathbf{C}_{1}^{\prime}\right)^{o}, \quad \mathbf{G}_{2}=\mathbf{G}_{1}\left(\mathbf{G}_{1}^{o} \mathbf{C}_{2}\right)^{o}, \quad \mathbf{G}_{3}=\mathbf{G}_{2}\left(\mathbf{G}_{2}^{\prime} \mathbf{C}_{3}^{\prime}\right)^{o}, \\
\mathbf{K}_{i} & =\mathbf{P}_{\Sigma^{1 / 2} G_{i-1}}-\mathbf{P}_{\Sigma^{1 / 2} G_{i}}, \quad i=1,2,3, \\
z_{11} & =\frac{v_{1}}{n-\operatorname{rank}\left(A_{1}\right)-v_{1}-1}, \quad z_{12}=\frac{k_{1} v_{2}}{n-\operatorname{rank}\left(A_{2}\right)-v_{2}-1}, \quad z_{22}=\frac{v_{2}}{n-\operatorname{rank}\left(A_{2}\right)-v_{2}-1}, \\
z_{13} & =\frac{k_{1} k_{2} v_{3}}{n-\operatorname{rank}\left(A_{3}\right)-v_{3}-1}, \quad z_{23}=\frac{v_{3}}{n-\operatorname{rank}\left(C_{3}\right)-v_{3}-1}, \quad z_{33}=\frac{\operatorname{rank}\left(A_{3}\right)-v_{3}-1}{n-}
\end{aligned}
$$

Open Access This article is distributed under the terms of the Creative Commons Attribution Noncommercial License which permits any noncommercial use, distribution, and reproduction in any medium, provided the original author(s) and source are credited.

\section{Appendix A: Multivariate beta distribution}

The following lemma is applied several times in the proof of Theorem 6 and the proof can be found in Kollo and von Rosen (2005) [Theorem 2.4.8, p. 248-250, Theorem 2.4 .15 , p. 263]. 
Lemma 1 Let $\mathbf{V} \sim W_{p}\left(\mathbf{I}_{p}, n\right), p<n$, and $\mathbf{W} \sim W_{p}\left(\mathbf{I}_{p}, m\right), p<m$. Then,

$$
\mathbf{B}=(\mathbf{V}+\mathbf{W})^{-1 / 2} \mathbf{V}(\mathbf{V}+\mathbf{W})^{-1 / 2}
$$

is multivariate beta type I distributed and $\mathbf{B}$ is independent of $\mathbf{V}+\mathbf{W}$. Moreover,

$$
\begin{aligned}
E[\mathbf{B}] & =\frac{n}{m+n} \mathbf{I}_{p}, \\
E\left[\mathbf{B}^{-1}\right] & =\frac{m+n-p-1}{n-p-1} \mathbf{I}_{p}, \quad n-p-1>0 .
\end{aligned}
$$

\section{Appendix B: Proof of Theorem 6 (iii)}

The proof of the Theorem 6 (iii) is very similar to the proof of Theorem 6 (ii) and therefore only a few details are given. From Theorem 1 it follows that

$$
\begin{aligned}
& \mathbf{K}\left(\widehat{\mathbf{B}}_{1}-\mathbf{B}_{1}\right) \mathbf{L}=\mathbf{K}\left(\mathbf{A}_{1}^{\prime} \mathbf{A}_{1}\right)^{-} \mathbf{A}_{1}^{\prime}(\mathbf{Y}-E[\mathbf{Y}]) \mathbf{S}_{3}^{-1} \mathbf{C}_{1}^{\prime}\left(\mathbf{C}_{1} \mathbf{S}_{3}^{-1} \mathbf{C}_{1}^{\prime}\right)^{-} \mathbf{L} \\
& -\mathbf{K}\left(\mathbf{A}_{1}^{\prime} \mathbf{A}_{1}\right)^{-} \mathbf{A}_{1}^{\prime} \mathbf{A}_{2}\left(\mathbf{A}_{2}^{\prime} \mathbf{Q}_{A_{1}} \mathbf{A}_{2}\right)^{-} \mathbf{A}_{2}^{\prime} \mathbf{Q}_{A_{1}}(\mathbf{Y}-E[\mathbf{Y}]) \mathbf{S}_{2}^{-1} \mathbf{C}_{2}^{\prime}\left(\mathbf{C}_{2} \mathbf{S}_{2}^{-1} \mathbf{C}_{2}^{\prime}\right)^{-} \mathbf{L} \\
& -\mathbf{K}\left(\mathbf{A}_{1}^{\prime} \mathbf{A}_{1}\right)^{-} \mathbf{A}_{1}^{\prime}\left(\mathbf{I}-\mathbf{A}_{2}\left(\mathbf{A}_{2}^{\prime} \mathbf{Q}_{A_{1}} \mathbf{A}_{2}\right)^{-} \mathbf{A}_{2}^{\prime} \mathbf{Q}_{A_{1}}\right) \mathbf{A}_{3}\left(\widehat{\mathbf{B}}_{3}-\mathbf{B}_{3}\right) \mathbf{C}_{3} \mathbf{C}_{1}^{\prime}\left(\mathbf{C}_{1} \mathbf{C}_{1}^{\prime}\right)^{-} \mathbf{L} .
\end{aligned}
$$

Since $\mathbf{A}_{1}^{\prime} \mathbf{Y}$ is independent of $\mathbf{S}_{1}, \mathbf{S}_{2}, \mathbf{S}_{3}, \mathbf{Q}_{A_{1}} \mathbf{Y}$ and $\widehat{\mathbf{B}}_{3}$, and $\mathbf{A}_{2}^{\prime} \mathbf{Q}_{A_{1}} \mathbf{Y}$ is independent of $\mathbf{S}_{1}, \mathbf{S}_{2}$ and $\widehat{\mathbf{B}}_{3}$ the terms given by (B-1), (B-2), (B-3) are uncorrelated. Thus,

$$
\begin{aligned}
D\left[\mathbf{K} \widehat{\mathbf{B}}_{1} \mathbf{L}\right]= & D\left[\mathbf{K}\left(\mathbf{A}_{1}^{\prime} \mathbf{A}_{1}\right)^{-} \mathbf{A}_{1}^{\prime}(\mathbf{Y}-E[\mathbf{Y}]) \mathbf{S}_{3}^{-1} \mathbf{C}_{1}^{\prime}\left(\mathbf{C}_{1} \mathbf{S}_{3}^{-1} \mathbf{C}_{1}^{\prime}\right)^{-} \mathbf{L}\right] \\
& +D\left[\mathbf{K}\left(\mathbf{A}_{1}^{\prime} \mathbf{A}_{1}\right)^{-} \mathbf{A}_{1}^{\prime} \mathbf{A}_{2}\left(\mathbf{A}_{2}^{\prime} \mathbf{Q}_{A_{1}} \mathbf{A}_{2}\right)^{-} \mathbf{A}_{2}^{\prime} \mathbf{Q}_{A_{1}}(\mathbf{Y}-E[\mathbf{Y}])\right. \\
& \left.\times \mathbf{S}_{2}^{-1} \mathbf{C}_{2}^{\prime}\left(\mathbf{C}_{2} \mathbf{S}_{2}^{-1} \mathbf{C}_{2}^{\prime}\right)^{-} \mathbf{L}\right] \\
& +D\left[\mathbf{K}\left(\mathbf{A}_{1}^{\prime} \mathbf{A}_{1}\right)^{-} \mathbf{A}_{1}^{\prime}\left(\mathbf{I}-\mathbf{A}_{2}\left(\mathbf{A}_{2}^{\prime} \mathbf{Q}_{A_{1}} \mathbf{A}_{2}\right)^{-} \mathbf{A}_{2}^{\prime} \mathbf{Q}_{A_{1}}\right) \mathbf{A}_{3}\left(\widehat{\mathbf{B}}_{3}-\mathbf{B}_{3}\right)\right. \\
& \left.\times \mathbf{C}_{3} \mathbf{C}_{1}^{\prime}\left(\mathbf{C}_{1} \mathbf{C}_{1}^{\prime}\right)^{-} \mathbf{L}\right]
\end{aligned}
$$

The dispersion in (B-6) is obtained from Theorem 6 (i) and (B-5) can be determined from the treatment of (10) via (11) and equality

$$
\left(\mathbf{A}_{1}^{\prime} \mathbf{A}_{1}\right)^{-} \mathbf{A}_{1}^{\prime} \mathbf{A}_{2}\left(\mathbf{A}_{2}^{\prime} \mathbf{Q}_{A_{1}} \mathbf{A}_{2}\right)^{-} \mathbf{A}_{2}^{\prime} \mathbf{A}_{1}\left(\mathbf{A}_{1}^{\prime} \mathbf{A}_{1}\right)^{-}=\left(\mathbf{A}_{1}^{\prime} \mathbf{Q}_{A_{2}} \mathbf{A}_{1}\right)^{-}-\left(\mathbf{A}_{1}^{\prime} \mathbf{A}_{1}\right)^{-}
$$

i.e., (B-5) equals

$$
\mathbf{L}^{\prime}\left(\mathbf{C}_{2} \mathbf{C}_{2}^{\prime}\right)^{-} \mathbf{C}_{2} \mathbf{F}_{1} \mathbf{C}_{2}^{\prime}\left(\mathbf{C}_{2} \mathbf{C}_{2}^{\prime}\right)^{-} \mathbf{L} \otimes \mathbf{K}\left[\left(\mathbf{A}_{1}^{\prime} \mathbf{Q}_{A_{2}} \mathbf{A}_{1}\right)^{-}-\left(\mathbf{A}_{1}^{\prime} \mathbf{A}_{1}\right)^{-}\right] \mathbf{K}^{\prime}
$$

where $\mathbf{F}_{1}$ is given by (22). Put

$$
\mathbf{F}_{2}=E\left[\mathbf{P}_{C_{1}^{\prime} ; S_{3}^{-1}} \boldsymbol{\Sigma} \mathbf{P}_{C_{1}^{\prime} ; S_{3}^{-1}}^{\prime}\right]
$$


and then (B-4) is determined through

$$
\mathbf{L}^{\prime}\left(\mathbf{C}_{1} \mathbf{C}_{1}^{\prime}\right)^{-} \mathbf{C}_{1} \mathbf{F}_{2} \mathbf{C}_{1}^{\prime}\left(\mathbf{C}_{1} \mathbf{C}_{1}^{\prime}\right)^{-} \mathbf{L} \otimes \mathbf{K}\left(\mathbf{A}_{1}^{\prime} \mathbf{A}_{1}\right)^{-} \mathbf{K}^{\prime}
$$

We will copy the approach for obtaining $\mathbf{F}_{1}$. Similarly as in (13)

$$
\mathbf{F}_{2}=\boldsymbol{\Sigma}-\boldsymbol{\Sigma}^{1 / 2} E\left[\mathbf{T}_{2}\right] \boldsymbol{\Sigma}^{1 / 2}-\boldsymbol{\Sigma}^{1 / 2} E\left[\mathbf{T}_{2}^{\prime}\right] \boldsymbol{\Sigma}^{1 / 2}+\boldsymbol{\Sigma}^{1 / 2} E\left[\mathbf{T}_{2} \mathbf{T}_{2}^{\prime}\right] \boldsymbol{\Sigma}^{1 / 2}
$$

where

$$
\begin{aligned}
& \mathbf{T}_{2}=\mathbf{V}_{3} \mathbf{D}_{1}^{o}\left(\mathbf{D}_{1}^{o^{\prime}} \mathbf{V}_{3} \mathbf{D}_{1}^{o}\right)^{-} \mathbf{D}_{1}^{o^{\prime}} \\
& \mathbf{D}_{1}^{o}=\boldsymbol{\Sigma}^{1 / 2} \mathbf{C}_{1}^{o}, \quad \mathbf{V}_{3}=\mathbf{V}_{2}+\mathbf{T}_{1} \mathbf{Z} \mathbf{P}_{Q_{A_{1}} A_{2}} \mathbf{Z}^{\prime} \mathbf{T}_{1}^{\prime}
\end{aligned}
$$

Moreover

$$
\mathbf{D}_{1}^{o^{\prime}} \mathbf{V}_{3} \mathbf{D}_{1}^{o}=\mathbf{D}_{1}^{o^{\prime}} \mathbf{W}_{2} \mathbf{D}_{1}^{o}+\mathbf{D}_{1}^{o^{\prime}} \mathbf{Z} \mathbf{P}_{Q_{A_{1}} A_{2}} \mathbf{Z}^{\prime} \mathbf{D}_{1}^{o} \equiv \mathbf{D}_{1}^{o^{\prime}} \mathbf{W}_{3} \mathbf{D}_{1}^{o}
$$

where $\mathbf{W}_{3} \sim W_{p}\left(\mathbf{I}_{p}, n-\operatorname{rank}\left(\mathbf{A}_{1}\right)\right)$. Because $\mathcal{R}\left(\mathbf{D}_{1}\right)^{\perp} \subseteq \mathcal{R}\left(\mathbf{D}_{2}\right)^{\perp} \subseteq \mathcal{R}\left(\mathbf{D}_{3}\right)^{\perp}$ we may write

$$
\mathbf{D}_{2}^{o^{\prime}} \mathbf{V}_{3} \mathbf{D}_{1}^{o}=\mathbf{D}_{2}^{o^{\prime}} \mathbf{W}_{3} \mathbf{D}_{1}^{o}, \quad \mathbf{D}_{1}^{o^{\prime}} \mathbf{V}_{3} \mathbf{D}_{1}^{o}=\mathbf{D}_{1}^{o^{\prime}} \mathbf{W}_{3} \mathbf{D}_{1}^{o}
$$

In correspondence with (14) we will study

$$
E\left[\mathbf{T}_{2}\right]=\mathbf{P}_{D_{2}^{o}} E\left[\mathbf{T}_{2}\right]+\mathbf{P}_{D_{3}} E\left[\mathbf{T}_{2}\right]+\mathbf{P}_{P_{D_{3}^{o}} D_{2}} E\left[\mathbf{T}_{2}\right]
$$

where

$$
\mathbf{P}_{P_{D_{3}^{o}} D_{2}}=\mathbf{D}_{3}^{o}\left(\mathbf{D}_{3}^{o^{\prime}} \mathbf{D}_{3}^{o}\right)^{-} \mathbf{D}_{3}^{o^{\prime}} \mathbf{D}_{2}\left(\mathbf{D}_{2}^{\prime} \mathbf{D}_{3}^{o}\left(\mathbf{D}_{3}^{o^{\prime}} \mathbf{D}_{3}^{o}\right)^{-} \mathbf{D}_{3}^{o^{\prime}} \mathbf{D}_{2}\right)^{-} \mathbf{D}_{2}^{\prime} \mathbf{D}_{3}^{o}\left(\mathbf{D}_{3}^{o^{\prime}} \mathbf{D}_{3}^{o}\right)^{-} \mathbf{D}_{3}^{o^{\prime}}
$$

is the orthogonal projection on $\mathcal{R}\left(\mathbf{D}_{3}\right)^{\perp} \cap \mathcal{R}\left(\mathbf{D}_{2}\right)$.

It follows that

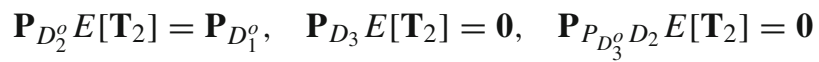

which implies $E\left[\mathbf{T}_{2}\right]=\mathbf{P}_{D_{1}^{o}}=\mathbf{I}_{p}-\mathbf{P}_{\Sigma^{-1 / 2} C_{1}^{\prime}}$.

Finally we will consider $E\left[\mathbf{T}_{2} \mathbf{T}_{2}^{\prime}\right]$. Now

$$
\mathbf{P}_{D_{2}^{o}} E\left[\mathbf{T}_{2} \mathbf{T}_{2}^{\prime}\right]\left(\mathbf{P}_{D_{3}}: \mathbf{P}_{P_{D_{3}^{o} D_{2}}}\right)=\mathbf{0}, \quad \mathbf{P}_{D_{3}} E\left[\mathbf{T}_{2} \mathbf{T}_{2}^{\prime}\right] \mathbf{P}_{P_{D_{3}^{o} D_{2}}}=\mathbf{0}
$$

and therefore it is enough to separately consider

$$
\mathbf{P}_{D_{2}^{o}} E\left[\mathbf{T}_{2} \mathbf{T}_{2}^{\prime}\right] \mathbf{P}_{D_{2}^{o}}, \quad \mathbf{P}_{D_{3}} E\left[\mathbf{T}_{2} \mathbf{T}_{2}^{\prime}\right] \mathbf{P}_{D_{3}}, \quad \mathbf{P}_{P_{D_{3}^{o}} D_{2}} E\left[\mathbf{T}_{2} \mathbf{T}_{2}^{\prime}\right] \mathbf{P}_{P_{D_{3}^{o}} D_{2}}
$$


First we observe that

$$
\mathbf{P}_{D_{2}^{o}} E\left[\mathbf{T}_{2} \mathbf{T}_{2}^{\prime}\right] \mathbf{P}_{D_{2}^{o}}=E\left[\mathbf{P}_{D_{2}^{o}} \mathbf{W}_{3} \mathbf{D}_{1}^{o}\left(\mathbf{D}_{1}^{o} \mathbf{W}_{3} \mathbf{D}_{1}^{o}\right)^{-} \mathbf{D}_{1}^{o^{\prime}} \mathbf{D}_{1}^{o}\left(\mathbf{D}_{1}^{o^{\prime}} \mathbf{W}_{3} \mathbf{D}_{1}^{o}\right)^{-} \mathbf{D}_{1}^{o^{\prime}} \mathbf{W}_{3} \mathbf{P}_{D_{2}^{o}}\right]
$$

which because of Wishartness of $\mathbf{W}_{3}$ equals (see also (17))

$$
\left(1-\gamma_{4}\right) \mathbf{P}_{D_{1}^{o}}+\gamma_{4} \mathbf{P}_{D_{2}^{o}}=\mathbf{I}_{p}-\left(1-\gamma_{4}\right) \mathbf{P}_{\Sigma^{-1 / 2} C_{1}^{\prime}}-\gamma_{4} \mathbf{P}_{\Sigma^{-1 / 2} C_{2}^{\prime}}
$$

where $\gamma_{4}$ was presented in the statement of the theorem. Moreover, we will use that

$$
\begin{aligned}
& \mathbf{V}_{2}^{-1}=\mathbf{V}_{2}^{-1} \mathbf{D}_{2}\left(\mathbf{D}_{2}^{\prime} \mathbf{V}_{2}^{-1} \mathbf{D}_{2}\right)^{-} \mathbf{D}_{2}^{\prime} \mathbf{V}_{2}^{-1}+\mathbf{D}_{2}^{o}\left(\mathbf{D}_{2}^{o^{\prime}} \mathbf{V}_{2} \mathbf{D}_{2}^{o}\right)^{-} \mathbf{D}_{2}^{o^{\prime}}, \\
& \mathbf{V}_{1}^{-1}=\mathbf{V}_{1}^{-1} \mathbf{D}_{3}\left(\mathbf{D}_{3}^{\prime} \mathbf{V}_{1}^{-1} \mathbf{D}_{3}\right)^{-} \mathbf{D}_{3}^{\prime} \mathbf{V}_{1}^{-1}+\mathbf{D}_{3}^{o}\left(\mathbf{D}_{3}^{o^{\prime}} \mathbf{V}_{1} \mathbf{D}_{3}^{o}\right)^{-} \mathbf{D}_{3}^{o^{\prime}}
\end{aligned}
$$

and then

$$
\begin{array}{rl}
\mathbf{P}_{D_{3}} & E\left[\mathbf{T}_{2} \mathbf{T}_{2}^{\prime}\right] \mathbf{P}_{D_{3}} \\
= & E\left[\operatorname{tr}\left\{\mathbf{V}_{3} \mathbf{D}_{1}^{o}\left(\mathbf{D}_{1}^{o^{\prime}} \mathbf{V}_{3} \mathbf{D}_{1}^{o}\right)^{-} \mathbf{D}_{1}^{o^{\prime}} \mathbf{D}_{1}^{o}\left(\mathbf{D}_{1}^{o^{\prime}} \mathbf{V}_{3} \mathbf{D}_{1}^{o}\right)^{-} \mathbf{D}_{1}^{o^{\prime}} \mathbf{V}_{3} \mathbf{V}_{1}^{-1}\right\}\right] \mathbf{P}_{D_{3}} \\
= & E\left[\operatorname{tr}\left\{\mathbf{V}_{2} \mathbf{V}_{2}^{-1} \mathbf{V}_{3} \mathbf{D}_{1}^{o}\left(\mathbf{D}_{1}^{o^{\prime}} \mathbf{V}_{3} \mathbf{D}_{1}^{o}\right)^{-} \mathbf{D}_{1}^{o^{\prime}} \mathbf{D}_{1}^{o}\left(\mathbf{D}_{1}^{o^{\prime}} \mathbf{V}_{3} \mathbf{D}_{1}^{o}\right)^{-} \mathbf{D}_{1}^{o^{\prime}} \mathbf{V}_{3} \mathbf{V}_{2}^{-1} \mathbf{V}_{2} \mathbf{V}_{1}^{-1}\right\}\right] \mathbf{P}_{D_{3}} \\
= & E\left[\operatorname { t r } \left\{\mathbf{V}_{2} \mathbf{D}_{2}^{o}\left(\mathbf{D}_{2}^{o^{\prime}} \mathbf{V}_{2} \mathbf{D}_{2}^{o}\right)^{-} \mathbf{D}_{2}^{o^{\prime}} \mathbf{V}_{3} \mathbf{D}_{1}^{o}\left(\mathbf{D}_{1}^{o^{\prime}} \mathbf{V}_{3} \mathbf{D}_{1}^{o}\right)^{-} \mathbf{D}_{1}^{o^{\prime}}\right.\right. \\
& \left.\left.\times \mathbf{D}_{1}^{o}\left(\mathbf{D}_{1}^{o^{\prime}} \mathbf{V}_{3} \mathbf{D}_{1}^{o}\right)^{-} \mathbf{D}_{1}^{o^{\prime}} \mathbf{V}_{3} \mathbf{D}_{2}^{o}\left(\mathbf{D}_{2}^{o^{\prime}} \mathbf{V}_{2} \mathbf{D}_{2}^{o}\right)^{-} \mathbf{D}_{2}^{o^{\prime}} \mathbf{V}_{2} \mathbf{V}_{1}^{-1}\right\}\right] \mathbf{P}_{D_{3}} \\
= & E\left[\operatorname { t r } \left\{\mathbf{D}_{3}^{o^{\prime}} \mathbf{W}_{2} \mathbf{D}_{2}^{o}\left(\mathbf{D}_{2}^{o^{\prime}} \mathbf{W}_{2} \mathbf{D}_{2}^{o}\right)^{-} \mathbf{D}_{2}^{o^{\prime}} \mathbf{W}_{3} \mathbf{D}_{1}^{o}\left(\mathbf{D}_{1}^{o^{\prime}} \mathbf{W}_{3} \mathbf{D}_{1}^{o}\right)^{-} \mathbf{D}_{1}^{o^{\prime}} \mathbf{D}_{1}^{o}\left(\mathbf{D}_{1}^{o^{\prime}} \mathbf{W}_{3} \mathbf{D}_{1}^{o}\right)^{-}\right.\right. \\
& \left.\left.\times \mathbf{D}_{1}^{o^{\prime}} \mathbf{W}_{3} \mathbf{D}_{2}^{o}\left(\mathbf{D}_{2}^{o^{\prime}} \mathbf{W}_{2} \mathbf{D}_{2}^{o}\right)^{-} \mathbf{D}_{2}^{o^{\prime}} \mathbf{W}_{2} \mathbf{D}_{3}^{o}\left(\mathbf{D}_{3}^{o^{\prime}} \mathbf{V}_{1} \mathbf{D}_{3}^{o}\right)^{-}\right\}\right] \mathbf{P}_{D_{3}} .
\end{array}
$$

By assumption $\mathcal{R}\left(\mathbf{D}_{1}\right)^{\perp} \subseteq \mathcal{R}\left(\mathbf{D}_{2}\right)^{\perp} \subseteq \mathcal{R}\left(\mathbf{D}_{3}\right)^{\perp}$ there exist matrices $\mathbf{U}_{1}$ and $\mathbf{U}_{2}$ such that

$$
\mathbf{D}_{1}^{o}=\mathbf{D}_{2}^{o} \mathbf{U}_{1}, \quad \mathbf{D}_{2}^{o}=\mathbf{D}_{3}^{o} \mathbf{U}_{2}
$$

Hence, (B-9) equals

$$
\begin{aligned}
& E\left[\operatorname { t r } \left\{\left(\mathbf{D}_{2}^{o^{\prime}} \mathbf{W}_{2} \mathbf{D}_{2}^{o}\right)^{-} \mathbf{D}_{2}^{o^{\prime}} \mathbf{W}_{3} \mathbf{D}_{1}^{o}\left(\mathbf{D}_{1}^{o^{\prime}} \mathbf{W}_{3} \mathbf{D}_{1}^{o}\right)^{-} \mathbf{D}_{1}^{o^{\prime}} \mathbf{D}_{1}^{o}\left(\mathbf{D}_{1}^{o^{\prime}} \mathbf{W}_{3} \mathbf{D}_{1}^{o}\right)^{-} \mathbf{D}_{1}^{o^{\prime}} \mathbf{W}_{3} \mathbf{D}_{2}^{o}\right.\right. \\
& \left.\left.\quad \times\left(\mathbf{D}_{2}^{o^{\prime}} \mathbf{W}_{2} \mathbf{D}_{2}^{o}\right)^{-} \mathbf{U}_{2}^{\prime} \mathbf{D}_{3}^{o^{\prime}} \mathbf{W}_{2} \mathbf{D}_{3}^{o}\left(\mathbf{D}_{3}^{o^{\prime}} \mathbf{V}_{1} \mathbf{D}_{3}^{o}\right)^{-} \mathbf{D}_{3}^{o^{\prime}} \mathbf{W}_{2} \mathbf{D}_{3}^{o} \mathbf{U}_{2}\right\}\right] \mathbf{P}_{D_{3}} .
\end{aligned}
$$

Furthermore, according to Lemma $1, \mathbf{N}_{1}=\left(\mathbf{D}_{3}^{o^{\prime}} \mathbf{W}_{2} \mathbf{D}_{3}^{o}\right)^{-1 / 2} \mathbf{D}_{3}^{o^{\prime}} \mathbf{V}_{1} \mathbf{D}_{3}^{o}\left(\mathbf{D}_{3}^{o^{\prime}} \mathbf{W}_{2} \mathbf{D}_{3}^{o}\right)^{-1 / 2}$ is independent of $\mathbf{D}_{3}^{o} \mathbf{W}_{2} \mathbf{D}_{3}^{o}$ and $\mathbf{D}_{1}^{o} \mathbf{W}_{3} \mathbf{D}_{1}^{o}$ and

$$
E\left[\mathbf{N}_{1}^{-1}\right]=\gamma_{5} \mathbf{I}_{p-\operatorname{rank}\left(C_{3}\right)}
$$


Similarly, $\mathbf{N}_{2}=\left(\mathbf{D}_{2}^{o} \mathbf{W}_{3} \mathbf{D}_{2}^{o}\right)^{-1 / 2} \mathbf{D}_{2}^{o} \mathbf{W}_{2} \mathbf{D}_{2}^{o}\left(\mathbf{D}_{2}^{o^{\prime}} \mathbf{W}_{3} \mathbf{D}_{2}^{o}\right)^{-1 / 2}$ is independent of $\mathbf{D}_{2}^{o^{\prime}} \mathbf{W}_{3} \mathbf{D}_{2}^{o}$,

$$
E\left[\mathbf{N}_{2}^{-1}\right]=\gamma_{6} \mathbf{I}_{p-\operatorname{rank}\left(C_{2}\right)}
$$

and $\mathbf{N}_{3}=\left(\mathbf{D}_{1}^{o^{\prime}} \mathbf{W}_{3} \mathbf{D}_{1}^{o}\right)^{-1 / 2} \mathbf{D}_{1}^{o^{\prime}} \mathbf{W}_{2} \mathbf{D}_{1}^{o}\left(\mathbf{D}_{1}^{o^{\prime}} \mathbf{W}_{3} \mathbf{D}_{1}^{o}\right)^{-1 / 2}$ is independent of $\mathbf{D}_{1}^{o^{\prime}} \mathbf{W}_{3} \mathbf{D}_{1}^{o}$,

$$
\begin{aligned}
E\left[\mathbf{N}_{3}\right] & =\gamma_{7} \mathbf{I}_{p-\operatorname{rank}\left(C_{1}\right)}, \\
E\left[\mathbf{N}_{3}^{-1}\right] & =\gamma_{8} \mathbf{I}_{p-\operatorname{rank}\left(C_{1}\right)} .
\end{aligned}
$$

Then, (B-10) equals

$$
\begin{aligned}
E[ & \operatorname{tr}\left\{\left(\mathbf{D}_{2}^{o^{\prime}} \mathbf{W}_{2} \mathbf{D}_{2}^{o}\right)^{-} \mathbf{D}_{2}^{o^{\prime}} \mathbf{W}_{3} \mathbf{D}_{1}^{o}\left(\mathbf{D}_{1}^{o^{\prime}} \mathbf{W}_{3} \mathbf{D}_{1}^{o}\right)^{-} \mathbf{D}_{1}^{o^{\prime}} \mathbf{D}_{1}^{o}\left(\mathbf{D}_{1}^{o^{\prime}} \mathbf{W}_{3} \mathbf{D}_{1}^{o}\right)^{-} \mathbf{D}_{1}^{o^{\prime}} \mathbf{W}_{3} \mathbf{D}_{2}^{o}\right. \\
& \left.\left.\times\left(\mathbf{D}_{2}^{o^{\prime}} \mathbf{W}_{2} \mathbf{D}_{2}^{o}\right)^{-} \mathbf{U}_{2}^{\prime}\left(\mathbf{D}_{3}^{o^{\prime}} \mathbf{W}_{2} \mathbf{D}_{3}^{o}\right)^{1 / 2} \mathbf{N}_{1}^{-1}\left(\mathbf{D}_{3}^{o^{\prime}} \mathbf{W}_{2} \mathbf{D}_{3}^{o}\right)^{1 / 2} \mathbf{U}_{2}\right\}\right] \mathbf{P}_{D_{3}} \\
= & \gamma_{5} E\left[\operatorname { t r } \left\{\mathbf{D}_{2}^{o^{\prime}} \mathbf{W}_{3} \mathbf{D}_{1}^{o}\left(\mathbf{D}_{1}^{o^{\prime}} \mathbf{W}_{3} \mathbf{D}_{1}^{o}\right)^{-} \mathbf{D}_{1}^{o^{\prime}} \mathbf{D}_{1}^{o}\left(\mathbf{D}_{1}^{o^{\prime}} \mathbf{W}_{3} \mathbf{D}_{1}^{o}\right)^{-} \mathbf{D}_{1}^{o^{\prime}} \mathbf{W}_{3} \mathbf{D}_{2}^{o}\right.\right. \\
& \left.\left.\times\left(\mathbf{D}_{2}^{o^{\prime}} \mathbf{W}_{2} \mathbf{D}_{2}^{o}\right)^{-}\right\}\right] \mathbf{P}_{D_{3}} \\
= & \gamma_{5} E\left[\operatorname { t r } \left\{\left(\mathbf{D}_{1}^{o^{\prime}} \mathbf{W}_{3} \mathbf{D}_{1}^{o}\right)^{-} \mathbf{D}_{1}^{o^{\prime}} \mathbf{D}_{1}^{o}\left(\mathbf{D}_{1}^{o^{\prime}} \mathbf{W}_{3} \mathbf{D}_{1}^{o}\right)^{-}\right.\right. \\
& \left.\left.\times \mathbf{U}_{1}^{\prime}\left(\mathbf{D}_{2}^{o^{\prime}} \mathbf{W}_{3} \mathbf{D}_{2}^{o}\right)^{1 / 2} \mathbf{N}_{2}^{-1}\left(\mathbf{D}_{2}^{o^{\prime}} \mathbf{W}_{3} \mathbf{D}_{2}^{o}\right)^{1 / 2} \mathbf{U}_{1}\right\}\right] \mathbf{P}_{D_{3}} \\
= & \gamma_{5} \gamma_{6} E\left[\operatorname{tr}\left\{\left(\mathbf{D}_{1}^{o^{\prime}} \mathbf{W}_{3} \mathbf{D}_{1}^{o}\right)^{-} \mathbf{D}_{1}^{o^{\prime}} \mathbf{D}_{1}^{o}\left(\mathbf{D}_{1}^{o^{\prime}} \mathbf{W}_{3} \mathbf{D}_{1}^{o}\right)^{-} \mathbf{D}_{1}^{o^{\prime}} \mathbf{W}_{3} \mathbf{D}_{1}^{o}\right\}\right] \mathbf{P}_{D_{3}} \\
= & \gamma_{5} \gamma_{6} E\left[\operatorname{tr}\left\{\mathbf{D}_{1}^{o}\left(\mathbf{D}_{1}^{o^{\prime}} \mathbf{W}_{3} \mathbf{D}_{1}^{o}\right)^{-} \mathbf{D}_{1}^{o^{\prime}}\right\}\right] \mathbf{P}_{D_{3}}=\gamma_{4} \gamma_{5} \gamma_{6} \mathbf{P}_{D_{3}} \\
= & \gamma_{4} \gamma_{5} \gamma_{6} \mathbf{P}_{\Sigma^{-1 / 2} C_{3}^{\prime} .}
\end{aligned}
$$

The last expression which will be considered requires also some calculations:

$$
\begin{aligned}
& \mathbf{P}_{P_{D_{3}^{o}} D_{2}} E\left[\mathbf{T}_{2} \mathbf{T}_{2}^{\prime}\right] \mathbf{P}_{P_{D_{3}^{o}} D_{2}} \\
& =E\left[\mathbf{P}_{P_{D_{3}^{o}} D_{2}} \mathbf{V}_{3} \mathbf{D}_{1}^{o}\left(\mathbf{D}_{1}^{o^{\prime}} \mathbf{W}_{3} \mathbf{D}_{1}^{o}\right)^{-} \mathbf{D}_{1}^{o^{\prime}} \mathbf{D}_{1}^{o}\left(\mathbf{D}_{1}^{o^{\prime}} \mathbf{W}_{3} \mathbf{D}_{1}^{o}\right)^{-} \mathbf{D}_{1}^{o^{\prime}} \mathbf{V}_{3} \mathbf{P}_{P_{D_{3}^{o} D_{2}}}\right] \\
& =E\left[\mathbf{P}_{P_{D_{3}^{o}} D_{2}} \mathbf{V}_{2} \mathbf{D}_{1}^{o}\left(\mathbf{D}_{1}^{o^{\prime}} \mathbf{W}_{3} \mathbf{D}_{1}^{o}\right)^{-} \mathbf{D}_{1}^{o^{\prime}} \mathbf{D}_{1}^{o}\left(\mathbf{D}_{1}^{o^{\prime}} \mathbf{W}_{3} \mathbf{D}_{1}^{o}\right)^{-} \mathbf{D}_{1}^{o^{\prime}} \mathbf{V}_{2} \mathbf{P}_{P_{D_{3}^{o}} D_{2}}\right] \\
& +E\left[\mathbf{P}_{P_{D_{3}^{o} D_{2}}} \mathbf{V}_{2} \mathbf{D}_{2}^{o}\left(\mathbf{D}_{2}^{o^{\prime}} \mathbf{W}_{2} \mathbf{D}_{2}^{o}\right)^{-} \mathbf{D}_{2}^{o^{\prime}}\left(\mathbf{W}_{3}-\mathbf{W}_{2}\right) \mathbf{D}_{1}^{o}\left(\mathbf{D}_{1}^{o^{\prime}} \mathbf{W}_{3} \mathbf{D}_{1}^{o}\right)^{-} \mathbf{D}_{1}^{o^{\prime}}\right. \\
& \left.\times \mathbf{D}_{1}^{o}\left(\mathbf{D}_{1}^{o^{\prime}} \mathbf{W}_{3} \mathbf{D}_{1}^{o}\right)^{-} \mathbf{D}_{1}^{o} \mathbf{V}_{2} \mathbf{P}_{P_{D_{3}^{o} D_{2}}}\right] \\
& +E\left[\mathbf{P}_{P_{D_{3}^{o}} D_{2}} \mathbf{V}_{2} \mathbf{D}_{1}^{o}\left(\mathbf{D}_{1}^{o^{\prime}} \mathbf{W}_{3} \mathbf{D}_{1}^{o}\right)^{-} \mathbf{D}_{1}^{o^{\prime}} \mathbf{D}_{1}^{o}\left(\mathbf{D}_{1}^{o^{\prime}} \mathbf{W}_{3} \mathbf{D}_{1}^{o}\right)^{-} \mathbf{D}_{1}^{o^{\prime}}\left(\mathbf{W}_{3}-\mathbf{W}_{2}\right)\right. \\
& \left.\times \mathbf{D}_{2}^{o}\left(\mathbf{D}_{2}^{o^{\prime}} \mathbf{W}_{2} \mathbf{D}_{2}^{o}\right)^{-} \mathbf{D}_{2}^{o^{\prime}} \mathbf{V}_{2} \mathbf{P}_{P_{D_{3}^{o}} D_{2}}\right] \\
& +E\left[\mathbf{P}_{P_{D_{3}^{o}} D_{2}} \mathbf{V}_{2} \mathbf{D}_{2}^{o}\left(\mathbf{D}_{2}^{o^{\prime}} \mathbf{W}_{2} \mathbf{D}_{2}^{o}\right)^{-} \mathbf{D}_{2}^{o^{\prime}}\left(\mathbf{W}_{3}-\mathbf{W}_{2}\right) \mathbf{D}_{1}^{o}\left(\mathbf{D}_{1}^{o^{\prime}} \mathbf{W}_{3} \mathbf{D}_{1}^{o}\right)^{-} \mathbf{D}_{1}^{o^{\prime}}\right. \\
& \left.\times \mathbf{D}_{1}^{o}\left(\mathbf{D}_{1}^{o^{\prime}} \mathbf{W}_{3} \mathbf{D}_{1}^{o}\right)^{-} \mathbf{D}_{1}^{o^{\prime}}\left(\mathbf{W}_{3}-\mathbf{W}_{2}\right) \mathbf{D}_{2}^{o}\left(\mathbf{D}_{2}^{o^{\prime}} \mathbf{W}_{2} \mathbf{D}_{2}^{o}\right)^{-} \mathbf{D}_{2}^{o^{\prime}} \mathbf{V}_{2} \mathbf{P}_{P_{D_{3}^{o}} D_{2}}\right] \text {. }
\end{aligned}
$$


Now (B-12) equals

$$
\begin{aligned}
E\left[\operatorname{tr}\left\{\mathbf{W}_{2} \mathbf{D}_{1}^{o}\left(\mathbf{D}_{1}^{o^{\prime}} \mathbf{W}_{3} \mathbf{D}_{1}^{o}\right)^{-} \mathbf{D}_{1}^{o^{\prime}} \mathbf{D}_{1}^{o}\left(\mathbf{D}_{1}^{o^{\prime}} \mathbf{W}_{3} \mathbf{D}_{1}^{o}\right)^{-} \mathbf{D}_{1}^{o^{\prime}}\right\}\right] \mathbf{P}_{P_{D_{3}^{o} D_{2}}} & =\gamma_{4} \gamma_{7} \mathbf{P}_{P_{D_{3}^{o}} D_{2}} \\
& =\gamma_{4} \gamma_{7} \mathbf{P}_{Q_{\Sigma^{-1 / 2} C_{3}^{\prime}}}{ }^{-1 / 2} C_{2}^{\prime}
\end{aligned}
$$

Turning to (B-13) this expression equals

$$
\begin{aligned}
E[ & \mathbf{P}_{P_{D_{3}^{o}} D_{2}} \mathbf{W}_{2} \mathbf{D}_{2}^{o}\left(\mathbf{D}_{2}^{o^{\prime}} \mathbf{W}_{2} \mathbf{D}_{2}^{o}\right)^{-} \mathbf{D}_{2}^{o^{\prime}}\left(\mathbf{W}_{3}-\mathbf{W}_{2}\right) \mathbf{D}_{1}^{o}\left(\mathbf{D}_{1}^{o^{\prime}} \mathbf{W}_{3} \mathbf{D}_{1}^{o}\right)^{-} \mathbf{D}_{1}^{o^{\prime}} \mathbf{D}_{1}^{o} \\
& \left.\times\left(\mathbf{D}_{1}^{o^{\prime}} \mathbf{W}_{3} \mathbf{D}_{1}^{o}\right)^{-} \mathbf{D}_{1}^{o^{\prime}} \mathbf{W}_{2} \mathbf{P}_{P_{D_{3}^{o}} D_{2}}\right] \\
= & E\left[\operatorname{tr}\left\{\left(\mathbf{W}_{3}-\mathbf{W}_{2}\right) \mathbf{D}_{1}^{o}\left(\mathbf{D}_{1}^{o^{\prime}} \mathbf{W}_{3} \mathbf{D}_{1}^{o}\right)^{-} \mathbf{D}_{1}^{o^{\prime}} \mathbf{D}_{1}^{o}\left(\mathbf{D}_{1}^{o^{\prime}} \mathbf{W}_{3} \mathbf{D}_{1}^{o}\right)^{-} \mathbf{D}_{1}^{o^{\prime}}\right\}\right] \mathbf{P}_{P_{D_{3}^{o} D_{2}}} \\
= & E\left[\operatorname{tr}\left\{\mathbf{D}_{1}^{o}\left(\mathbf{D}_{1}^{o^{\prime}} \mathbf{W}_{3} \mathbf{D}_{1}^{o}\right)^{-} \mathbf{D}_{1}^{o^{\prime}}\right\}\right. \\
& \left.-\operatorname{tr}\left\{\mathbf{D}_{1}^{o}\left(\mathbf{D}_{1}^{o^{\prime}} \mathbf{W}_{3} \mathbf{D}_{1}^{o}\right)^{-} \mathbf{D}_{1}^{o^{\prime}} \mathbf{W}_{2} \mathbf{D}_{1}^{o}\left(\mathbf{D}_{1}^{o^{\prime}} \mathbf{W}_{3} \mathbf{D}_{1}^{o}\right)^{-} \mathbf{D}_{1}^{o^{\prime}}\right\}\right] \mathbf{P}_{P_{D_{3}^{o}} D_{2}} \\
= & \gamma_{4}\left(1-\gamma_{7}\right) \mathbf{P}_{P_{D_{3}^{o}} D_{2}}=\gamma_{4}\left(1-\gamma_{7}\right) \mathbf{P}_{Q_{\Sigma^{-1 / 2} C_{3}^{\prime}} \Sigma^{-1 / 2} C_{2}^{\prime}}
\end{aligned}
$$

By symmetry we obtain the same expression for (B-14). Finally it is observed that (B-15) equals

$$
\begin{aligned}
& E\left[\mathbf{P}_{P_{D_{3}^{o}} D_{2}} \mathbf{W}_{2} \mathbf{D}_{2}^{o}\left(\mathbf{D}_{2}^{o^{\prime}} \mathbf{W}_{2} \mathbf{D}_{2}^{o}\right)^{-} \mathbf{D}_{2}^{o^{\prime}}\left(\mathbf{W}_{3}-\mathbf{W}_{2}\right) \mathbf{D}_{1}^{o}\left(\mathbf{D}_{1}^{o^{\prime}} \mathbf{W}_{3} \mathbf{D}_{1}^{o}\right)^{-} \mathbf{D}_{1}^{o^{\prime}} \mathbf{D}_{1}^{o}\right. \\
& \left.\times\left(\mathbf{D}_{1}^{o^{\prime}} \mathbf{W}_{3} \mathbf{D}_{1}^{o}\right)^{-} \mathbf{D}_{1}^{o^{\prime}}\left(\mathbf{W}_{3}-\mathbf{W}_{2}\right) \mathbf{D}_{2}^{o}\left(\mathbf{D}_{2}^{o^{\prime}} \mathbf{W}_{2} \mathbf{D}_{2}^{o}\right)^{-} \mathbf{D}_{2}^{o^{\prime}} \mathbf{W}_{2} \mathbf{P}_{P_{D_{3}^{o}} D_{2}}\right] \\
& =E\left[\operatorname { t r } \left\{\mathbf{W}_{2} \mathbf{D}_{2}^{o}\left(\mathbf{D}_{2}^{o^{\prime}} \mathbf{W}_{2} \mathbf{D}_{2}^{o}\right)^{-} \mathbf{D}_{2}^{o^{\prime}}\left(\mathbf{W}_{3}-\mathbf{W}_{2}\right) \mathbf{D}_{1}^{o}\left(\mathbf{D}_{1}^{o^{\prime}} \mathbf{W}_{3} \mathbf{D}_{1}^{o}\right)^{-} \mathbf{D}_{1}^{o^{\prime}}\right.\right. \\
& \left.\left.\times \mathbf{D}_{1}^{o}\left(\mathbf{D}_{1}^{o^{\prime}} \mathbf{W}_{3} \mathbf{D}_{1}^{o}\right)^{-} \mathbf{D}_{1}^{o^{\prime}}\left(\mathbf{W}_{3}-\mathbf{W}_{2}\right) \mathbf{D}_{2}^{o}\left(\mathbf{D}_{2}^{o^{\prime}} \mathbf{W}_{2} \mathbf{D}_{2}^{o}\right)^{-} \mathbf{D}_{2}^{o^{\prime}}\right\}\right] \mathbf{P}_{P_{D_{3}^{o}} D_{2}} \\
& =E\left[\operatorname { t r } \left\{\mathbf{W}_{3} \mathbf{D}_{1}^{o}\left(\mathbf{D}_{1}^{o} \mathbf{W}_{3} \mathbf{D}_{1}^{o}\right)^{-} \mathbf{D}_{1}^{o} \mathbf{D}_{1}^{o}\left(\mathbf{D}_{1}^{o} \mathbf{W}_{3} \mathbf{D}_{1}^{o}\right)^{-} \mathbf{D}_{1}^{o^{\prime}} \mathbf{W}_{3} \mathbf{D}_{2}^{o}\right.\right. \\
& \left.\left.\times\left(\mathbf{D}_{2}^{o^{\prime}} \mathbf{W}_{2} \mathbf{D}_{2}^{o}\right)^{-} \mathbf{D}_{2}^{o^{\prime}}\right\}\right] \mathbf{P}_{P_{D_{3}^{o}} D_{2}} \\
& -2 E\left[\operatorname{tr}\left\{\mathbf{W}_{3} \mathbf{D}_{1}^{o}\left(\mathbf{D}_{1}^{o^{\prime}} \mathbf{W}_{3} \mathbf{D}_{1}^{o}\right)^{-} \mathbf{D}_{1}^{o^{\prime}} \mathbf{D}_{1}^{o}\left(\mathbf{D}_{1}^{o^{\prime}} \mathbf{W}_{3} \mathbf{D}_{1}^{o}\right)^{-} \mathbf{D}_{1}^{o^{\prime}}\right\}\right] \mathbf{P}_{P_{D_{3}^{o}} D_{2}} \\
& +E\left[\operatorname{tr}\left\{\mathbf{W}_{2} \mathbf{D}_{1}^{o}\left(\mathbf{D}_{1}^{o^{\prime}} \mathbf{W}_{3} \mathbf{D}_{1}^{o}\right)^{-} \mathbf{D}_{1}^{o^{\prime}} \mathbf{D}_{1}^{o}\left(\mathbf{D}_{1}^{o^{\prime}} \mathbf{W}_{3} \mathbf{D}_{1}^{o}\right)^{-} \mathbf{D}_{1}^{o^{\prime}}\right\}\right] \mathbf{P}_{P_{D_{3}^{o} D_{2}}} \\
& =E\left[\operatorname { t r } \left\{\left(\mathbf{D}_{1}^{o^{\prime}} \mathbf{W}_{3} \mathbf{D}_{1}^{o}\right)^{-} \mathbf{D}_{1}^{o^{\prime}} \mathbf{D}_{1}^{o}\left(\mathbf{D}_{1}^{o^{\prime}} \mathbf{W}_{3} \mathbf{D}_{1}^{o}\right)^{-}\right.\right. \\
& \left.\left.\times \mathbf{U}_{1}^{\prime}\left(\mathbf{D}_{2}^{o^{\prime}} \mathbf{W}_{3} \mathbf{D}_{2}^{o}\right)^{1 / 2} \mathbf{N}_{2}^{-1}\left(\mathbf{D}_{2}^{o^{\prime}} \mathbf{W}_{3} \mathbf{D}_{2}^{o}\right)^{1 / 2} \mathbf{U}_{1}\right\}\right] \mathbf{P}_{P_{D_{3}^{o}} D_{2}} \\
& -2 E\left[\operatorname{tr}\left\{\mathbf{D}_{1}^{o}\left(\mathbf{D}_{1}^{o^{\prime}} \mathbf{W}_{3} \mathbf{D}_{1}^{o}\right)^{-} \mathbf{D}_{1}^{o^{\prime}}\right\}\right] \mathbf{P}_{P_{D_{3}^{o} D_{2}}} \\
& +E\left[\operatorname{tr}\left\{\left(\mathbf{D}_{1}^{o^{\prime}} \mathbf{W}_{3} \mathbf{D}_{1}^{o}\right)^{-1 / 2} \mathbf{D}_{1}^{o^{\prime}} \mathbf{D}_{1}^{o}\left(\mathbf{D}_{1}^{o^{\prime}} \mathbf{W}_{3} \mathbf{D}_{1}^{o}\right)^{-1 / 2} \mathbf{N}_{3}\right\}\right] \mathbf{P}_{P_{D_{3}^{o}} D_{2}} \\
& =\left(\gamma_{6}-2+\gamma_{7}\right) E\left[\operatorname{tr}\left\{\mathbf{D}_{1}^{o}\left(\mathbf{D}_{1}^{o^{\prime}} \mathbf{W}_{3} \mathbf{D}_{1}^{o}\right)^{-} \mathbf{D}_{1}^{o^{\prime}}\right\}\right] \mathbf{P}_{P_{D_{3}^{o} D_{2}}} \\
& =\gamma_{4}\left(\gamma_{6}-2+\gamma_{7}\right) \mathbf{P}_{P_{D_{3}^{o}} D_{2}}=\gamma_{4}\left(\gamma_{6}-2+\gamma_{7}\right) \mathbf{P}_{Q_{\Sigma^{-1 / 2} C_{3}^{\prime}} \Sigma^{-1 / 2} C_{2}^{\prime}} \text {. }
\end{aligned}
$$


Thus, summing (B-8), (B-11), (B-16), (B-17) and (B-18) we obtain

$$
\begin{array}{r}
E\left[\mathbf{T}_{2} \mathbf{T}_{2}^{\prime}\right]=\left(1-\gamma_{4}\right)\left(\mathbf{I}_{p}-\mathbf{P}_{\Sigma^{-1 / 2} C_{1}^{\prime}}\right)+\gamma_{4}\left(\mathbf{I}_{p}-\mathbf{P}_{\Sigma^{-1 / 2} C_{2}^{\prime}}\right) \\
+\gamma_{4} \gamma_{5} \gamma_{6} \mathbf{P}_{\Sigma^{-1 / 2} C_{3}^{\prime}}+\gamma_{4} \gamma_{6} \mathbf{P}_{Q_{\Sigma^{-1 / 2} C_{3}^{\prime}} \Sigma^{-1 / 2} C_{2}^{\prime}}
\end{array}
$$

and then

$$
\begin{gathered}
\mathbf{F}_{2}=\Sigma^{1 / 2}\left[\mathbf{I}-\left(1+\gamma_{4}\right) \mathbf{P}_{\Sigma^{-1 / 2} C_{1}^{\prime}}+\gamma_{4} \mathbf{P}_{\Sigma^{-1 / 2} C_{2}^{\prime}}+\gamma_{4} \gamma_{5} \gamma_{6} \mathbf{P}_{\Sigma^{-1 / 2} C_{3}^{\prime}}\right. \\
+\gamma_{4} \gamma_{6} \mathbf{P}_{\left.Q_{\Sigma^{-1 / 2} C_{3}^{\prime}} \Sigma^{-1 / 2} C_{2}^{\prime}\right] \Sigma^{1 / 2} .} .
\end{gathered}
$$

From this and (B-4)-(B-6) we obtain the theorem.

\section{References}

Andersson SA, Perlman MD (1993) Lattice models for conditional independence in a multivariate normal distribution. Ann Statist 22:1318-1358

Andersson SA, Perlman MD (1998) Normal linear regression models with recursive graphical Markov structure. J Multivariate Anal 66:133-187

Baksalary JK (1984) A study of the equivalence between a Gauss-Markoff model and its augmentation by nuisance parameters. Math Operationsforsch u Stat 15:3-35

Druilhet P (1999) Optimality of circular neighbor balanced designs. J Stat Plann Infer 81:141-152

Filipiak K, Markiewicz A, Szczepańska A (2009) Optimal designs under a multivariate linear model with additional nuisance parameters. Stat Papers 50:761-778

Filipiak K, Różański R (2009) Connectedness of complete block designs under an interference model. Stat Papers 50:779-787

Kollo T, von Rosen D (2005) Advanced multivariate statistics with matrices. Springer, Dordrecht

Kunert J (1983) Optimal design and refinement of the linear model with applications to repeated measurements designs. Ann Stat 11:247-257

Kunert J, Martin RJ (2000) On the determination of optimal designs for an interference model. Ann Stat 28:1728-1742

Markiewicz A (2001) On dependence structure preserving optimality. Stat Probab Lett 53:415-419

Markiewicz A, Szczepańska A (2007) Optimal designs in multivariate linear models. Stat Probab Lett 77:426-430

Potthof RF, Roy SN (1964) A generalized multivariate analysis of variance model useful especially for growth curve problems. Biometrika 51:313-326

Verbyla AP, Venables WN (1988) An extension of the growth curve model. Biometrika 75:129-138 